JOURNAL OF THE

AMERICAN MATHEMATICAL SOCIETY

Volume 22, Number 3, July 2009, Pages 607-639

S 0894-0347(09)00629-8

Article electronically published on February 6, 2009

\title{
SINGULAR KÄHLER-EINSTEIN METRICS
}

\author{
PHILIPPE EYSSIDIEUX, VINCENT GUEDJ, AND AHMED ZERIAHI
}

\section{INTRODUCTION}

Thirty years ago, in a celebrated article [Y, S. T. Yau (and independently T. Aubin [A] ) solved the Calabi conjecture by studying complex Monge-Ampère equations on a compact Kähler manifold.

Since then, complex Monge-Ampère equations have been extremely useful in Kähler geometry (see for instance DP]) and in the dynamical study of rational mappings (see $[S]$ and references therein).

Two major developments in the theory of complex Monge-Ampère equations occurred in the last decade. In the local theory, a deeper analysis of the image of the complex Monge-Ampère operator [Ce], [K1] has followed the pioneering work of E. Bedford and B. A. Taylor $[\mathrm{BT}$. In the global theory a new proof of the $\mathcal{C}^{0}$-estimate [K1], K2] has allowed one to treat complex Monge-Ampère equations with more degenerate R.H.S.

In [GZ1], GZ2], two of us revisited and extended the results of [BT], Ce on complex Monge-Ampère operators to compact Kähler manifolds. In the present article, we use these methods to study complex Monge-Ampère equations with degenerate L.H.S. We first define, in the spirit of [Ce, GZ2, weak solutions to degenerate complex Monge-Ampère equations and then prove, using ideas of [K1], K2 , that these solutions are bounded:

Theorem A. Let $X$ be a compact Kähler manifold, $\omega$ a semi-positive (1,1)-form such that $\int_{X} \omega^{n}>0$ and $0 \leq f \in L^{p}\left(X, \omega^{n}\right), p>1$, a density such that $\int_{X} f \omega^{n}=$ $\int_{X} \omega^{n}$. Then there is a unique bounded function $\varphi$ on $X$ such that $\omega+d d^{c} \varphi \geq 0$ and

$$
\left(\omega+d d^{c} \varphi\right)^{n}=f \omega^{n} \quad \text { with } \sup _{X} \varphi=-1 .
$$

Furthermore, $\varphi$ is continuous provided there exists a decreasing sequence $\varphi_{j}$ of continuous $\omega$-psh functions with $\lim \varphi_{j}=\varphi$ and $f \mapsto \varphi$ is a continuous map from $L^{p}\left(X, \omega^{n}\right)$ to $L^{\infty}(X)$.

When $\omega$ has algebraic singularities, then $\mu$ can be assumed to have $L^{p}$ density $(p>1)$ with respect to the Lebesgue measure.

When for every $\omega$-psh $\varphi$ there is a decreasing sequence of continuous $\omega$-psh functions converging to $\varphi$ pointwise, we will say $(X,[\omega])$ enjoys the continuous approximation property. We believe it holds in great generality but the problem turned out to be more subtle than expected; hence we leave this as an open problem for further research.

Received by the editors March 17, 2006.

2000 Mathematics Subject Classification. Primary 32W20, 32Q20, 32J27, 14J17.

(C)2009 American Mathematical Society Reverts to public domain 28 years from publication 
With this $L^{\infty}$-estimate, it is possible to adapt classical ideas of [Y] and [Ts and prove

Theorem B. Let $X$ be projective algebraic complex manifold, $\omega$ a smooth semipositive closed $(1,1)$-form that is positive outside a complex subvariety $S \subset X$. Let $\Omega$ be a Kähler form on $X$. Assume furthermore that $\omega^{n}=D \Omega^{n}$ where $D^{-\epsilon}$ is in $L^{1}\left(\Omega^{n}\right)$ and that $[\omega],[\Omega] \in N S_{\mathbb{R}}(X)$.

Let $\sigma_{1}, \ldots, \sigma_{p}$ (resp. $\tau_{1}, \ldots, \tau_{q}$ ) be holomorphic sections of some line bundle (resp. of some other line bundle). Assume $k, l \in \mathbb{R}_{\geq 0}$ and $F \in \mathcal{C}^{\infty}(X, \mathbb{R})$ are fixed so that

$$
\int_{X} \frac{1}{\left|\tau_{1}\right|^{2 l}+\ldots+\left|\tau_{q}\right|^{2 l}} \Omega^{n}<\infty \text { and } \int_{X} \frac{\left|\sigma_{1}\right|^{2 k}+\ldots+\left|\sigma_{p}\right|^{2 k}}{\left|\tau_{1}\right|^{2 l}+\ldots+\left|\tau_{q}\right|^{2 l}} e^{F} \Omega^{n}=\int_{X} \omega^{n} .
$$

Then the unique bounded function $\varphi$ such that $\omega+d d^{c} \varphi \geq 0$ and

$$
\left(\omega+d d^{c} \varphi\right)^{n}=\frac{\left|\sigma_{1}\right|^{2 k}+\ldots+\left|\sigma_{p}\right|^{2 k}}{\left|\tau_{1}\right|^{2 l}+\ldots+\left|\tau_{q}\right|^{2 l}} e^{F} \Omega^{n}, \text { with } \sup _{X} \varphi=-1,
$$

is smooth outside $B=S \cup \bigcap_{i}\left\{\sigma_{i}=0\right\} \cup \bigcap_{i}\left\{\tau_{i}=0\right\}$.

This result should be compared with $[\mathrm{Y}$, Theorem 8, p. 403. Yau's result is stronger in many respects, most notably in the absence of any projectivity/rationality assumption and a more precise regularity theory. On the other hand, the condition on the poles of the L.H.S. is less optimal than here. We expect the projectivity/rationality assumptions to be superfluous.

Observe that the condition on the singularity in the L.H.S. is precisely the condition that the singular metric associated to $l\left(\tau_{i}\right)$ has a trivial multiplier ideal sheaf, or if $q=1$, that the pair $\left(X, l\left(\tau_{1}\right)\right)$ is klt (see Definition 6.7 ). The possibility of solving complex Monge-Ampère equations with $L^{p}$-R.H.S. was first established by S. Kolodziej K1, K2, and the connection with the singularities of the Minimal Model Program (MMP for short) has been a strong incentive to our work.

From an algebraic geometer's perspective, these results may be viewed as a version of $[\mathbf{Y}]$ for normal Kähler spaces.

As a by-product, S. T. Yau constructed Kähler-Einstein metrics on smooth canonically polarized manifolds and Ricci-flat metrics on what is now known as Calabi-Yau manifolds. It had been soon realized [Kö] that this also yields KählerEinstein metrics on Kähler orbifolds, hence on the canonical models of surfaces of general type, since they have isolated quotient singularities.

In higher dimension, in spite of the development of the MMP during the 1980sculminating with $\mathrm{Mo}$ and the proof of the existence of canonical models for general type 3-folds $\mathrm{Ka}$ - there was no satisfying analog of these Kähler-Einstein metrics.

For smooth minimal general type projective manifolds, H. Tsuji proved Ts that an appropriate Kähler-Ricci flow starting with an arbitrary Kähler datum exists in infinite time, converges towards a current representing the canonical class which is smooth outside the exceptional locus of the map to the canonical model, and defines a Kähler-Einstein metric ther 1 . The conjecture made there that the current has continuous (or even bounded) local potentials partly motivated our work.

The article [Ts has been revisited in the recent preprint [CN] and in [TZ] (we learned of them when finishing the present work), where a very satisfactory proof of convergence towards a current with bounded potentials is given. The independent

\footnotetext{
${ }^{1}$ Although his idea was rather compelling, the details of the proof for convergence were somewhat hard to follow.
} 
work [TZ] uses a slightly weaker version of Theorem A and does not give any detail on the proof. These details have been indeed provided subsequently in [Zha]. On the other hand, the three approaches tend to emphasize different aspects of the problem and seem to complement each other nicely.

In this article we give a more general theory of singular Kähler-Einstein metrics as a consequence of the following result:

Theorem C. Let $(V, \Delta)$ be a projective klt pair such that $K_{V}+\Delta$ is an ample $\mathbb{Q}$-divisor. Then there is a unique semi-Kähler current in $\left[K_{V}+\Delta\right]$ with bounded potentials, which satisfies a global degenerate Monge-Ampère equation on $V$ and defines a smooth Kähler-Einstein metric of negative curvature on $(V-\Delta)^{\text {reg }}$.

Let $(V, \Delta)$ be a projective klt pair such that $K_{V}+\Delta \cong 0$ (Q্-linear equivalence of $\mathbb{Q}$-Cartier divisors). Then in every ample class in $N S_{\mathbb{R}}(V)$ there is a unique semi-Kähler current with bounded potentials, which satisfies a global degenerate Monge-Ampère equation on $V$ and defines a Ricci flat metric on $(V-\Delta)^{\text {reg }}$.

The precise formulation and Monge-Ampère equations are to be found in Theorems 7.5 and 7.8 below.

A large part of the MMP is now confirmed to work in higher dimension. Based on ideas of [Sho, developped further on by $\mathrm{HMcK}$, the preprint [BCHM] has achieved a full proof of the finite generation of the canonical ring for any projective manifold. Using this, we obtain

Corollary D. Let $X$ be a projective manifold of general type and let $V=X_{\text {can }}$ be the unique model of $X$ with only canonical singularities and $K_{V}$ ample. Then $K_{V}$ contains a unique singular Kähler-Einstein metric $\omega_{K E}$ of negative curvature.

Note that we do not assume that the singularities are quotient singularities nor that $X$ has a smooth minimal model (a strong restriction present in [Ts], TZ], [CN]). On the other hand if $\pi: X \rightarrow X_{c a n}$ is a resolution of singularities, then $\left[\pi^{*} \omega_{K E}\right] \cong K_{X}+F$ where $F \geq 0$ and $=0$ iff $\pi$ is crepant and $X$ is a smooth minimal model.

The problem of constructing a singular Kähler-Einstein metric on a canonically polarized projective variety with canonical singularities $X_{\text {can }}$ had already been considered in $[\mathrm{Sg} 2$, with a different approach. Theorem 5.6 in $[\mathrm{Sg}$ there and its proof imply that given $\pi: X \rightarrow X_{\text {can }}$ a log-resolution, there is a closed positive current $T_{K E}$ in $\pi^{*} K_{X_{\text {can }}}$ with zero Lelong numbers such that $T_{K E}$ is smooth on $\pi^{-1}\left(X_{c a n}^{r e g}\right)$ and defines a KE metric there. This construction agrees with ours, and our contribution is that $T_{K E}$ has a bounded potential coming from $X_{c a n}$.

One may also try to construct these singular KE metrics unconditionnally in an approach to the finiteness of the canonical ring [Siu2]. Needless to say, it is a substantially harder task.

Let us state yet another corollary:

Corollary E. Let $X$ be a projective variety with only canonical singularities such that $K_{X} \sim_{\mathbb{Q}} 0$ and let $A \in N S_{\mathbb{R}}(X)$ be an ample class. Then $A$ contains a unique singular Ricci-flat metric $\omega_{C Y}$ with bounded potentials.

The local potentials of $\omega_{C Y}$ are continuous provided $(V, A)$ satisfies the continuous approximation property.

${ }^{2}$ We apologize for having overlooked this reference in the first circulated version of this work. 
For the applications, the regularity theory of singular Kähler-Einstein metrics still needs to be developped more thoroughly.

\section{NOTATION AND ORGANIZATION OF THE PAPER}

In the entire paper, $X$ will denote a compact Kähler manifold of dimension $n$, $\omega$ a smooth closed form of bidegree $(1,1)$ which is non-negative and big, i.e. the smooth measure $\omega^{n}$ is not identical to zero. For convenience we normalize $\omega$ so that

$$
\operatorname{Vol}_{\omega}(X):=\int_{X} \omega^{n}=1 .
$$

$V$ will denote a normal complex space. A resolution of $V$ will be a locally projective bimeromorphic holomorphic morphism $\pi: X \rightarrow V, X$ being smooth, such that $\pi: \pi^{-1}\left(V^{r e g}\right) \rightarrow V^{r e g}$ is an isomorphism. A resolution $\pi$ is a logresolution iff $\pi^{-1}\left(V^{\text {sing }}\right)$ is a divisor with simple normal crossings. Assume we have a coherent ideal sheaf $\mathcal{I} \subset \mathcal{O}_{V}$. A log-resolution of $(V, \mathcal{I})$ is a projective bimeromorphic holomorphic morphism $\pi: X \rightarrow V, X$ being smooth, such that $\pi: \pi^{-1}\left((V-Z(\mathcal{I}))^{r e g}\right) \rightarrow(V-Z(\mathcal{I}))^{r e g}$ is an isomorphism with the additional property that the ideal sheaf $\pi^{-1} \mathcal{I}$. $\mathcal{O}_{X} 3$ satisfies $\pi^{-1} \mathcal{I} \cdot \mathcal{O}_{X}=\mathcal{O}_{X}\left(-\sum \gamma_{E} E\right) \subset \mathcal{O}_{X}$ where $\gamma_{E} \in \mathbb{N}$ is a positive integer attached to an exceptional divisor $E$ of $\pi$.

A pair is a pair $(V, \Delta)$ with $V$ a normal complex space and $\Delta$ a $\mathbb{Q}$-Weil divisor $\Delta=\sum_{i} d_{i} E_{i}$ where $0 \leq d_{i} \leq 1$ are rational numbers and $\left(E_{i}\right)_{i}$ is a finite family of pairwise distinct irreducible codimension 1 subvarieties of $V$. A log-resolution of a pair is a log-resolution of the ideal $\mathcal{I}_{N \Delta}$ where $N$ is an integer such that $N d_{i} \in \mathbb{N} 4$

All these flavors of log-resolutions exist by [Hi], BM if the variety (resp. pair) under consideration is open in (resp. a restriction to an open subset of) a compact variety (pair). The resolution can then be assumed to be a projective morphism.

The paper is organized as follows. In Section 1 we define, following [GZ2, the set $\mathcal{E}^{1}(X, \omega)$ of $\omega$-psh functions with finite self-energy and produce weak solutions to complex Monge-Ampère equations $\left(\omega+d d^{c} \varphi\right)^{n}=\mu$ in the class $\mathcal{E}^{1}(X, \omega)$ (see Proposition 1.4). This is our first basic observation: weak solutions are easy to produce in $\mathcal{E}^{1}(X, \omega)$.

The continuity of the solutions is studied in Section 2 (see Theorem 2.1) by using ideas from [K1], [K2], [K3] and GZ1]. This, together with Propositions 3.1 and 3.3, yields Theorem A. We actually expect the solutions to be Hölder-continuous, as Theorem 3.5 indicates. We indeed establish further regularity results in Section 3, especially Theorem 3.6, by using ideas of $[\mathrm{Y}], \mathrm{Ts}$. This yields Theorem B.

In Section 4 we solve Monge-Ampère equations of the type $\left(\omega+d d^{c} \varphi\right)^{n}=e^{t \varphi} \mu$, $t>0$ (see Theorem 4.1 and Proposition 4.4) by a fixed point method. Here again the use of class $\mathcal{E}^{1}(X, \omega)$ makes life easier and allows us to reduce our analysis to previously studied Monge-Ampère equations $\left(\omega+d d^{c} \varphi\right)^{n}=\mu^{\prime}$.

\footnotetext{
${ }^{3} \pi^{-1} \mathcal{I} \cdot \mathcal{O}_{X}$ is locallly the ideal sheaf of $\mathcal{O}_{X}$ generated by the family of holomorphic functions $\left(\pi^{*} f_{I}\right)_{I}$, where $\left(f_{I}\right)_{I}$ are local generators of $\mathcal{I}$. The set $Z(\mathcal{I})$ is the analytic subvariety defined by $\mathcal{I}$.

${ }^{4}$ The MMP is conjectured to work for $\mathbb{Q}$-factorial dlt pairs provided $V$ is projective algebraic. This seemingly technical extension of the MMP is known as log-MMP. log-MMP works in dimension $\leq 3$. The philosophy of the log-MMP is to define the canonical divisor of a pair to be $K_{(V, \Delta)}:=K_{V}+\Delta$ and to try to prove the same theorems for pairs and for varieties. See BCHM] for the strongest results in this direction.
} 
In Section 5 we recall some basic facts on some of the singularities encountered in the MMP, and in Section 6 we explain what sort of measures $\mu$ we need to consider in order to produce Kähler-Einstein metrics. An important observation is Lemma 6.4, which shows that it is necessary to restrict to the case of log-terminal singularities (see Definition 5.3).

In section 7 we show how our results from Sections 2, 3, 4 allow us to produce singular Kähler-Einstein metrics (see Theorems 7.5, 7.8, 7.12). This is where we prove Theorem C.

\section{Weak solutions to Monge-Ampère equations}

In this section $\omega$ will denote a smooth semi-positive closed $(1,1)$-form which is big, i.e. satisfies $\int_{X} \omega^{n}>0$. For any Kähler form $\Omega$ on $X$ and for all $\varepsilon>0$, the form $\omega_{\varepsilon}:=\omega+\varepsilon \Omega$ is again Kähler on $X$.

We are going to extend several results that are known to hold true when $\omega$ is Kähler to the present more general setting.

Recall that the set of $\omega$-plurisubharmonic functions ( $\omega$-psh for short) is

$$
P S H(X, \omega):=\left\{\varphi \in L^{1}(X, \mathbb{R} \cup\{-\infty\}) / d d^{c} \varphi \geq-\omega \text { and } \varphi \text { is u.s.c. }\right\} \text {. }
$$

We refer the reader to GZ1 for basic properties of $\omega$-psh functions. The following subclass has been extensively studied in GZ2]:

Definition 1.1. We let $\mathcal{E}^{1}(X, \omega)$ denote the set of $\omega$-psh functions with finite selfenergy: this is the set of functions $\varphi \in P S H(X, \omega)$ for which there exists a sequence $\varphi_{j} \in P S H(X, \omega) \cap L^{\infty}(X)$ such that

$$
\varphi_{j} \searrow \varphi \text { and } \sup _{j} \int_{X}\left(-\varphi_{j}\right)\left(\omega+d d^{c} \varphi_{j}\right)^{n}<+\infty .
$$

This class of functions is studied in GZ2 when $\omega$ is a Kähler form. We leave it to the reader to check that the basic properties of this class of functions proved in GZ2 when $\omega$ is Kähler apply with no modification to the present case. In particular the complex Monge-Ampère operator $\left(\omega+d d^{c} \varphi\right)^{n}$ is well-defined for $\varphi \in \mathcal{E}^{1}(X, \omega)$, and it is continuous on decreasing sequences of functions in $\mathcal{E}^{1}(X, \omega)$.

We shall need a slightly more general continuity result, which takes into account the dependence in $\omega$ :

Proposition 1.2. Fix $\Omega$ a Kähler form on $X$, and let $\left(\varepsilon_{j}\right)$ be a sequence of positive real numbers decreasing to zero. Let $\varphi_{j} \in \mathcal{E}^{1}\left(X, \omega+\varepsilon_{j} \Omega\right)$ be a sequence of functions which decrease pointwise towards $\varphi$ and such that

$$
\sup _{j \geq 1} \int_{X}\left|\varphi_{j}\right|\left(\omega+\varepsilon_{j} \Omega+d d^{c} \varphi_{j}\right)^{n}<+\infty .
$$

Then $\varphi \in \mathcal{E}^{1}(X, \omega)$, and $\left(\omega+\varepsilon_{j} \Omega+d d^{c} \varphi_{j}\right)^{n} \rightarrow\left(\omega+d d^{c} \varphi\right)^{n}$.

Proof. Set $\omega_{j}:=\omega+\varepsilon_{j} \Omega$. We can assume w.l.o.g. that $\varphi \leq \varphi_{j} \leq 0$. Set

$$
\varphi^{K}:=\max (\varphi,-K) \in P S H(X, \omega) \text { and } \varphi_{j}^{K}:=\max \left(\varphi_{j},-K\right) \in P S H\left(X, \omega_{j}\right) .
$$

Observe that, $K$ being fixed, $\left(\varphi_{j}^{K}\right)_{j}$ is uniformly bounded and decreases towards $\varphi^{K}$ as $j$ goes to infinity. Therefore $\left(\omega_{j}+d d^{c} \varphi_{j}^{K}\right)^{n} \rightarrow\left(\omega+d d^{c} \varphi^{K}\right)^{n}$, by a classical 
result of E. Bedford and A. Taylor [BT]. Moreover the sequence of positive measures $\left(-\varphi_{j}^{K}\right)\left(\omega_{j}+d d^{c} \varphi_{j}^{K}\right)^{n}$ has uniformly bounded mass, since by Lemma 2.3 in GZ2,

$$
0 \leq \int_{X}\left(-\varphi_{j}^{K}\right)\left(\omega_{j}+d d^{c} \varphi_{j}^{K}\right)^{n} \leq 2^{n} \int_{X}\left(-\varphi_{j}\right)\left(\omega_{j}+d d^{c} \varphi_{j}\right)^{n} \leq 2^{n} M,
$$

where $M:=\sup _{j} \int_{X}\left(-\varphi_{j}\right)\left(\omega_{j}+d d^{c} \varphi_{j}\right)^{n}<+\infty$.

Since $\varphi_{j}$ is u.s.c., a standard argument yields that any cluster point $\nu$ of the sequence $\left(-\varphi_{j}^{K}\right)\left(\omega_{j}+d d^{c} \varphi_{j}^{K}\right)^{n}$ satisfies $0 \leq\left(-\varphi^{K}\right)\left(\omega+d d^{c} \varphi^{K}\right)^{n} \leq \nu$. In particular

$$
0 \leq \int_{X}\left(-\varphi^{K}\right)\left(\omega+d d^{c} \varphi^{K}\right)^{n} \leq \liminf _{j \rightarrow+\infty} \int\left(-\varphi_{j}^{K}\right)\left(\omega_{j}+d d^{c} \varphi_{j}^{K}\right)^{n} \leq 2^{n} M
$$

is bounded from above uniformly with respect to $K$. Since $\varphi^{K}$ decreases towards $\varphi$, this shows $\varphi \in \mathcal{E}^{1}(X, \omega)$.

It remains to show that $\left(\omega_{j}+d d^{c} \varphi_{j}\right)^{n} \rightarrow\left(\omega+d d^{c} \varphi\right)^{n}$. Since $\left(\omega_{j}+d d^{c} \varphi_{j}^{K}\right)^{n} \rightarrow$ $\left(\omega+d d^{c} \varphi^{K}\right)^{n}$ for any fixed $K$, it is enough to get an upper bound on the mass of $\left(\omega_{j}+d d^{c} \varphi_{j}^{K}\right)^{n}$ in $\left(\varphi_{j} \leq-K\right)$ which is uniform in $j$. This follows from Chebyshev inequality, namely

$$
\int_{\left(\varphi_{j} \leq-K\right)}\left(\omega_{j}+d d^{c} \varphi_{j}\right)^{n} \leq \frac{1}{K} \int_{X}\left(-\varphi_{j}\right)\left(\omega_{j}+d d^{c} \varphi_{j}\right)^{n} \leq \frac{M}{K} .
$$

This yields the desired result.

The Monge-Ampère capacity $\operatorname{Cap}_{\omega}(\cdot)$ has been studied in [GZ1],

$$
\operatorname{Cap}_{\omega}(K):=\sup \left\{\int_{K} \omega_{u}^{n} / u \in P S H(X, \omega), 0 \leq u \leq 1\right\},
$$

where $K$ is a Borel subset of $X$. Here - and in the sequel-we use the notation $\omega_{u}:=\omega+d d^{c} u \geq 0$. In this article we are interested in measures which are dominated by the Monge-Ampère capacity in the following way:

Definition 1.3. A probability measure $\mu$ on $X$ satisfies condition $\mathcal{H}(\alpha, A, \omega)$ if for all Borel subset $K$ of $X$,

$$
\mu(K) \leq \operatorname{ACap}_{\omega}(K)^{1+\alpha} .
$$

It has been shown by S. Kolodziej that when $\omega$ is Kähler, a probability measure $\mu$ which satisfies $\mathcal{H}(\alpha, A, \omega)$ can be written as the Monge-Ampère measure of some continuous $\omega$-psh function. This is still true when $\omega$ is merely semi-positive and big, and the proof will occupy us until the end of Section 2. We start by observingfollowing GZ2 - that $\mu$ is the Monge-Ampère of a function $\varphi$ which is not too singular.

Proposition 1.4. Let $\mu$ be a probability measure on $X$ which satisfies condition $\mathcal{H}(\alpha, A, \omega)$. Then there exists a unique function $\varphi \in \mathcal{E}^{1}(X, \omega)$ s.t.

$$
\mu=\left(\omega+d d^{c} \varphi\right)^{n} \text { and } \sup _{X} \varphi=-1 .
$$

Proof. Fix $\Omega$ a Kähler form on $X$, and set $\omega_{j}:=\omega+\varepsilon_{j} \Omega$, where $\varepsilon_{j}>0$ decreases to 0 . We start by showing that $\mathcal{E}^{1}\left(X, \omega_{j}\right) \subset L^{1}(\mu)$.

Fix $\varphi \in \mathcal{E}^{1}\left(X, \omega_{j}\right)$. We can assume without loss of generality that $\sup _{X} \varphi=-1$. It follows from Propositions 3.6 and 2.7 in GZ1 that there exists a constant $C=$ 
$C(\omega, \Omega)>0$ independent of $j$ such that $\operatorname{Cap}_{\omega_{j}}(\varphi<-t) \leq C / t$ for all $t>0$. Since $\operatorname{Cap}_{\omega}(\cdot) \leq \operatorname{Cap}_{\omega_{j}}(\cdot)$, the measure $\mu$ satisfies $\mathcal{H}\left(\alpha, A, \omega_{j}\right)$. We infer

$$
0 \leq \int_{X}(-\varphi) d \mu=\int_{t=1}^{+\infty} \mu(\varphi<-t) d t \leq \frac{A C^{1+\alpha}}{\alpha}<+\infty,
$$

with an upper bound which is independent of $j$.

The main result in GZ2 guarantees in this case that there exists a unique function $\varphi_{j} \in \mathcal{E}^{1}\left(X, \omega_{j}\right)$ such that

$$
\left(\omega_{j}+d d^{c} \varphi_{j}\right)^{n}=\lambda_{j} \mu \text { and } \sup _{X} \varphi_{j}=-1,
$$

where $\lambda_{j}=\int_{X}\left(\omega+\varepsilon_{j} \Omega\right)^{n}>1$ decreases to 1 as $j$ goes to infinity.

The normalization $\sup _{X} \varphi_{j}=-1$ implies that the sequence $\left(\varphi_{j}\right)$ is relatively compact in $L^{1}(X)$ (see Proposition 2.7 in GZ1]). Let $\varphi$ be a cluster point of $\left(\varphi_{j}\right)$. Relabelling if necessary, we assume $\varphi_{j} \rightarrow \varphi$ in $L^{1}(X)$. Note that $\varphi \in P S H(X, \omega)$ and $\sup _{X} \varphi=-1$ (by Hartogs' lemma; see Proposition 2.7, GZ1]). We are going to show that $\varphi \in \mathcal{E}^{1}(X, \omega)$ and $\omega_{\varphi}^{n}=\mu$.

Set $\Phi_{j}:=\left(\sup _{l \geq j} \varphi_{l}\right)^{*}$, where $u^{*}$ denotes the upper-semi-continuous regularization of $u$. Then $\Phi_{j} \in \operatorname{PSH}\left(X, \omega_{j}\right)$ with $\Phi_{j} \geq \varphi_{j}$, hence $\Phi_{j} \in \mathcal{E}^{1}\left(X, \omega_{j}\right)$ (see Proposition 3.2 in GZ2]), and $\Phi_{j}$ decreases towards $\varphi$. For $l \geq j$, we have

$$
\left(\omega_{j}+d d^{c} \varphi_{l}\right)^{n} \geq\left(\omega_{l}+d d^{c} \varphi_{l}\right)^{n}=\lambda_{l} \mu \geq \mu .
$$

It follows therefore from an inequality due to J. -P. Demailly Dem1 that

$$
\left(\omega_{j}+d d^{c} \Phi_{j}\right)^{n} \geq \mu .
$$

Now by (1) and Lemma 2.3 in GZ2,

$$
0 \leq \int_{X}\left(-\Phi_{j}\right)\left(\omega_{j}+d d^{c} \Phi_{j}\right)^{n} \leq 2^{n} \int_{X}\left(-\varphi_{j}\right)\left(\omega_{j}+d d^{c} \varphi_{j}\right)^{n}=2^{n} \lambda_{j} \int_{X}\left(-\varphi_{j}\right) d \mu
$$

is uniformly bounded with respect to $j$ thanks to (1).

We infer from Proposition 1.2 that $\varphi \in \mathcal{E}^{1}(X, \omega)$ and $\left(\omega_{j}+d d^{c} \Phi_{j}\right)^{n} \rightarrow$ $\left(\omega+d d^{c} \varphi\right)^{n}$. Thus $\left(\omega+d d^{c} \varphi\right)^{n} \geq \mu$, but these are two probability measures, whence $\mu=\left(\omega+d d^{c} \varphi\right)^{n}$. The uniqueness of $\varphi$ follows from Theorem 3.3 in GZ2.

\section{Continuous solutions}

The goal of this section is to prove the following result:

Theorem 2.1. Let $\mu$ be a probability measure on $X$ which satisfies condition $\mathcal{H}(\alpha, A, \omega)$. Then there exists a unique bounded function $\varphi \in P S H(X, \omega)$ such that

$$
\mu=\left(\omega+d d^{c} \varphi\right)^{n} \text { and } \sup _{X} \varphi=-1 .
$$

Moreover $\|\varphi\|_{L^{\infty}(X)} \leq C$, where $C$ only depends on $\alpha, A$ and $\omega$.

Furthermore $\varphi$ is continuous provided $(X,[\omega])$ satisfies the continuous approximation property.

Definition 2.2. Let $X$ be a compact Kähler manifold and $\omega$ a smooth semi-positive closed $(1,1)$-form. We say $(X,[\omega])$ satisfies the continuous approximation property if for every $\omega$-psh bounded function $\varphi$ there is a decreasing sequence of continuous $\omega$-psh functions such that $\lim \varphi_{j}=\varphi$ pointwise. 
It follows from Demailly's regularisation theorem that if $X$ is smooth and [ $\omega]$ Kähler, then $(X,[\omega])$ satisfies the continuous approximation property; see [BK] for a simple proof.

The authors believe the approximation property is satisfied if $X$ is a resolution of singularities of a normal projective variety with canonical singularities and $[\omega] \in$ $N S(X)$ is the pull-back of an ample $A$ class of $V$. If so, one says that $(V, A)$ enjoys the continuous approximation property.

It follows from Proposition 1.4 that we already know the existence of a unique solution $\varphi \in \mathcal{E}^{1}(X, \omega)$ to this Monge-Ampère equation. We need to show it is continuous. The following result is the key to everything to follow.

Lemma 2.3. Let $\varphi, \psi \in \mathcal{E}^{1}(X, \omega)$ be two negative functions. Then for all $s>0$ and $0 \leq t \leq 1$

$$
t^{n} \operatorname{Cap}_{\omega}(\varphi-\psi<-s-t) \leq \int_{(\varphi-\psi<-s-t \psi)} \omega_{\varphi}^{n} .
$$

Proof. Fix $u \in P S H(X, \omega)$ with $0 \leq u \leq 1$. For $\delta>0$ we set $t=\delta /(1+\delta)$. Observe that $0 \leq t \leq 1$ and

$$
\{\varphi-\psi<-s-t\} \subset\left\{\varphi<\frac{\psi+\delta u}{1+\delta}-s-t\right\} \subset\{\varphi-\psi<-s-t \psi\} .
$$

Set $\tilde{\varphi}:=(\psi+\delta u) /(1+\delta)-s-t \in P S H(X, \omega)$. Observe that

$$
t^{n} \int_{(\varphi-\psi<-s-t)} \omega_{u}^{n} \leq \int_{(\varphi-\psi<-s-t)}\left[\frac{1}{1+\delta} \omega_{\psi}+\frac{\delta}{1+\delta} \omega_{u}\right]^{n} \leq \int_{(\varphi<\tilde{\varphi})}\left[\omega+d d^{c} \tilde{\varphi}\right]^{n} .
$$

It follows from the comparison principle in class $\mathcal{E}^{1}(X, \omega)$ that

$$
\int_{(\varphi<\tilde{\varphi})}\left[\omega+d d^{c} \tilde{\varphi}\right]^{n} \leq \int_{(\varphi<\tilde{\varphi})}\left[\omega+d d^{c} \varphi\right]^{n} \leq \int_{(\varphi-\psi<-s-t \psi)}\left[\omega+d d^{c} \varphi\right]^{n} .
$$

Taking the supremum over all $u$ 's yields the desired result.

We will also need the following elementary observation:

Lemma 2.4. Let $f: \mathbb{R}^{+} \rightarrow \mathbb{R}^{+}$be a decreasing right-continuous function such that $\lim _{+\infty} f=0$. Assume there exists $\alpha, B>0$ such that $f$ satisfies

$$
(H(\alpha, B)) \quad t f(s+t) \leq B[f(s)]^{1+\alpha}, \forall s>0, \forall 0 \leq t \leq 1 .
$$

Then there exists $S_{\infty}=S_{\infty}(\alpha, B) \in \mathbb{R}^{+}$such that $f(s)=0$ for all $s \geq S_{\infty}$.

Proof. Fix $s_{0}>0$ large enough so that $f\left(s_{0}\right)^{\alpha}<1 / 2 B$. We define a sequence $\left(s_{j}\right) \in \mathbb{R}_{+}^{\mathbb{N}}$ by induction in the following way. If $f\left(s_{0}\right)=0$, we stop here; otherwise we set

$$
s_{1}:=\sup \left\{s>s_{0} / f(s)>\frac{1}{2} f\left(s_{0}\right)\right\} .
$$

Observe that $s_{1} \leq 1+s_{0}$ thanks to $(H(\alpha, B))$ and by definition of $s_{0}$.

Since $f$ is right-continuous, we get $f\left(s_{1}\right) \leq f\left(s_{0}\right) / 2$. If $f\left(s_{1}\right)=0$, we stop here; otherwise we go on by induction, setting

$$
s_{j+1}:=\sup \left\{s>s_{j} / f(s)>\frac{1}{2} f\left(s_{j}\right)\right\} \text {. }
$$


At each step $f\left(s_{j+1}\right) \leq f\left(s_{j}\right) / 2$ and $s_{j+1} \leq 1+s_{j}$. However the sequence $\left(s_{j}\right)$ does not grow too fast. It follows indeed from $(H(\alpha, B))$ that if $s \in] s_{j}, s_{j+1}[$,

$$
\left(s-s_{j}\right) f(s) \leq B f\left(s_{j}\right)^{1+\alpha} \leq 2 B f(s) f\left(s_{j}\right)^{\alpha},
$$

since $f\left(s_{j}\right) / 2 \leq f(s) \leq f\left(s_{j}\right)$ on the interval $\left[s_{j}, s_{j+1}\right]$. We infer

$$
s_{j+1}-s_{j} \leq 2 B f\left(s_{j}\right)^{\alpha} \leq 2 B 2^{-j \alpha} f\left(s_{0}\right)^{\alpha} \leq 2^{-j \alpha} .
$$

Thus the sequence $\left(s_{j}\right)$ is bounded from above, with limit

$$
S_{\infty}=s_{0}+\sum_{j \geq 0}\left(s_{j+1}-s_{j}\right) \leq s_{0}+\frac{2 B f\left(s_{0}\right)^{\alpha}}{1-2^{-\alpha}} \leq s_{0}+\frac{1}{1-2^{-\alpha}} .
$$

Remarks 2.5. Observe that the starting time $s_{0}(f, \alpha, B)$ is invariant under dilatation $f \mapsto \lambda f$, which transforms $B$ into $B / \lambda^{\alpha}$. Note also that if $f(0)^{\alpha}<1 / 2 B$, then we can take $s_{0}=0$; hence we get in this case

$$
S_{\infty} \leq \frac{2 B}{1-2^{-\alpha}}[f(0)]^{\alpha} .
$$

To see how previous lemmas can be used, we first prove that the unique solution $\varphi \in \mathcal{E}^{1}(X, \omega)$ given by Proposition 1.4 is bounded.

A uniform bound on the solution. Let $\varphi \in \mathcal{E}^{1}(X, \omega)$ be the unique function such that $\mu=\left(\omega+d d^{c} \varphi\right)^{n}$ and $\sup _{X} \varphi=-1$. Set

$$
f(s):=\left[\operatorname{Cap}_{\omega}(\varphi<-s)\right]^{1 / n} .
$$

Observe that $f: \mathbb{R}^{+} \rightarrow \mathbb{R}^{+}$is a right-continuous decreasing function with $\lim _{+\infty} f=$ 0 . Since $\mu=\omega_{\varphi}^{n}$ satisfies $\mathcal{H}(\alpha, A, \omega)$, it follows from Lemma 2.2 applied to the function $\psi \equiv 0$ that $f$ satisfies $(H(\alpha, B))$ with $B=A^{1 / n}$.

It follows from Propositions 2.7 and 3.6 in GZ1 that $f(s) \leq C_{1} / s^{1 / n}$ for some constant $C_{1}$ which only depends on $\omega$. We can thus take a starting time $s_{0}=$ $2^{n / \alpha} C_{1}^{n} A^{1 / \alpha}$ (see the proof of Lemma 2.3) and get $f(s)=0$ for $s \geq S_{\infty}:=s_{0}+$ $\left(1-2^{-\alpha}\right)^{-1}$ : this shows that the sets $(\varphi<-s)$ are empty if $s \geq S_{\infty}$; hence

$$
\|\varphi\|_{L^{\infty}(X)} \leq 2^{n / \alpha} C_{1}^{n} A^{1 / \alpha}+\frac{1}{1-2^{-\alpha}} .
$$

We are now going to use a refinement of the previous reasoning in order to show that $\varphi$ is actually continuous if the continuous approximation property holds.

Proposition 2.6. Let $\varphi, \psi \in \mathcal{E}^{1}(X, \omega)$ be two functions such that $\sup _{X} \varphi=$ $\sup _{X} \psi=-1$ and fix $\varepsilon>0$. Assume $\omega_{\varphi}^{n}=\mu$ satisfies $\mathcal{H}(\alpha, A, \omega)$ and $\psi$ is bounded. There exists $C=C\left(\alpha, A, \omega,\|\psi\|_{L^{\infty}(X)}\right)>0$ such that

$$
\sup _{X}(\psi-\varphi) \leq \varepsilon+C\left[\operatorname{Cap}_{\omega}(\varphi-\psi<-\varepsilon)\right]^{\alpha / n} .
$$

This inequality can be interpreted as follows. Assume $\psi$ is also a solution to a Monge-Ampère equation $\omega_{\psi}^{n}=\mu^{\prime}$, where $\mu^{\prime}$ also satisfies $\mathcal{H}(\alpha, A, \omega)$. Then we can interchange the roles of $\varphi$ and $\psi$ and get an upper bound on $\|\varphi-\psi\|_{L^{\infty}(X)}$. The proposition then tells us that if $\varphi$ and $\psi$ are close in capacity, they are close in $L^{\infty}$-norm. 
Proof. Set $M:=\|\psi\|_{L^{\infty}(X)}$. Observe that when $t \geq 0,(\varphi-\psi<-s-t \psi) \subset$ $(\varphi-\psi<-s+t M)$; hence it follows from Lemma 2.3 that for all $s>0,0 \leq t \leq 1$,

$$
t^{n} \operatorname{Cap}_{\omega}(\varphi-\psi<-s-t) \leq(1+M)^{n} \int_{(\varphi-\psi<-s)} \omega_{\varphi}^{n},
$$

using the obvious substitutions $s \mapsto s-M t, t \mapsto(1+M) t$. Since $\mu=\omega_{\varphi}^{n}$ satisfies $\mathcal{H}(\alpha, A, \omega)$, we infer

$$
t^{n} \operatorname{Cap}_{\omega}(\varphi-\psi<-s-t) \leq A(1+M)^{n} \operatorname{Cap}_{\omega}(\varphi-\psi<-s)^{1+\alpha} .
$$

Consider

$$
f(s):=\left[\operatorname{Cap}_{\omega}(\varphi-\psi<-s-\varepsilon)\right]^{1 / n}, \quad s>0 .
$$

Then $f$ satisfies the condition $(H(\alpha, B))$ of Lemma 2.3 with $B=(1+M) A^{1 / n}$. Assume $f(0)=\left[\operatorname{Cap}_{\omega}(\varphi-\psi<-\varepsilon)\right]^{1 / n}<\frac{1}{(2 B)^{1 / \alpha}}$. It follows in this case from Remarks 2.5 that $f(s)=0$ for $s \geq S_{\infty}$, where

$$
S_{\infty} \leq \frac{2 B}{1-2^{-\alpha}}\left[\operatorname{Cap}_{\omega}(\varphi-\psi<-\varepsilon)\right]^{\alpha / n} .
$$

Therefore the sets $\{\varphi-\psi<-s-\varepsilon\}$ are empty for $s>S_{\infty}$; hence

$$
\sup _{X}(\psi-\varphi) \leq \varepsilon+S_{\infty} \leq \varepsilon+\frac{2(1+M) A^{1 / n}}{1-2^{-\alpha}}\left[\operatorname{Cap}_{\omega}(\varphi-\psi<-\varepsilon)\right]^{\alpha / n} .
$$

Thus we can take here $C \geq 2 B /\left(1-2^{-\alpha}\right)$.

$$
\begin{aligned}
& \text { If } f(0)=\left[\operatorname{Cap}_{\omega}(\varphi-\psi<-\varepsilon)\right]^{1 / n} \geq(2 B)^{-1 / \alpha} \text {, then } \\
& \qquad \sup _{X}(\psi-\varphi) \leq-\inf _{X} \varphi \leq C_{2}(\alpha, A, \omega),
\end{aligned}
$$

by (2); hence it suffices to take $C \geq 2 B C_{2}$ to conclude.

Proof of Theorem 2.1. Let $\varphi \in \mathcal{E}^{1}(X, \omega)$ be the unique solution to the normalized Monge-Ampère equation $\mu=\left(\omega+d d^{c} \varphi\right)^{n}, \sup _{X} \varphi=-1$ (see Proposition 1.4). It follows from (2) that $\varphi$ is bounded.

If $(X,[\omega])$ enjoys the continuous approximation property, let $\varphi_{j}$ be a decreasing sequence of $\omega$-psh functions decreasing pointwise to $\varphi$. Since $\sup _{X} \varphi=-1$, we can assume $\varphi_{j} \leq 0$. Since $\varphi$ is bounded and $\varphi_{j} \geq \varphi$, the functions $\varphi_{j}$ are uniformly bounded on $X$.

Observe that $\varphi_{j}$ converges towards $\varphi$ in capacity (see Proposition 3.7 in [GZ1]); hence $\lim \operatorname{Cap}_{\omega}\left(\varphi-\varphi_{j}<-\varepsilon\right)=0$, for all $\varepsilon>0$. It follows therefore from Proposition 2.6 (applied with $\psi=\varphi_{j}$ ) that for all $\varepsilon>0$,

$$
\lim _{j \rightarrow+\infty}\left\|\varphi-\varphi_{j}\right\|_{L^{\infty}(X)}=\lim _{j \rightarrow+\infty} \sup _{X}\left(\varphi_{j}-\varphi\right) \leq \varepsilon .
$$

Thus $\left(\varphi_{j}\right)$ converges uniformly towards $\varphi$; hence $\varphi$ is continuous.

\section{More Regularity}

3.1. Measures with density. We now turn to the study of the complex MongeAmpère equation

$$
\left(\omega+d d^{c} \varphi\right)^{n}=\mu,
$$

when $\mu=f \omega^{n}$ is a measure with density $0 \leq f \in L^{p}\left(\omega^{n}\right), p>1$. 
Proposition 3.1. Assume $\mu=f \omega^{n}$ is a probability measure with density $0 \leq f \in$ $L^{p}(X)$, for some $p>1$. Then for any $\alpha>0$, there exists $A_{\alpha}>0$ such that $\mu$ satisfies $\mathcal{H}\left(\alpha, A_{\alpha}, \omega\right)$.

Proof. It is enough to establish $\mathcal{H}\left(\alpha, A_{\alpha}, \omega\right)$ for compact subsets, by regularity of $\mu$ and $C_{a p}$. Let $K$ be a compact subset of $X$. It follows from Hölder's inequality that

$$
0 \leq \mu(K) \leq\|f\|_{L^{p}\left(\omega^{n}\right)}\left[\operatorname{Vol}_{\omega}(K)\right]^{1 / q},
$$

where $1 / p+1 / q=1$. Note that $\|f\|_{L^{p}\left(\omega^{n}\right)}=1$ since we assume $\mu$ is a probability measure. We claim that

$$
\operatorname{Vol}_{\omega}(K) \leq C_{\omega} \exp \left[-\gamma_{\omega}\left(\operatorname{Cap}_{\omega}(K)\right)^{-1 / n}\right]
$$

for some constants $C_{\omega}, \gamma_{\omega}>0$ that only depend on $\omega$. We will be done if we can prove (4) since we can then check by elementary computations that $\exp \left(-x^{-\delta}\right)$ is dominated from above by $A_{\alpha} x^{\alpha}$, for all $x \in[0,1]$.

The set of functions $\mathcal{F}_{0}:=\left\{\varphi \in P S H(X, \omega) / \sup _{X} \varphi=0\right\}$ is compact in $L^{1}(X)$ (see Proposition 2.7 in [GZ1]). These functions have Lelong numbers $\nu(\varphi, x) \leq \nu_{\omega}$ bounded from above by a uniform constant. It follows therefore from Skoda's uniform integrability theorem [ Z that

$$
\sup _{\varphi \in \mathcal{F}_{0}} \int \exp \left[-\frac{2 \varphi}{\nu_{\omega}+1}\right] \omega^{n} \leq C_{2}<+\infty
$$

Set $\gamma_{\omega}:=2 /\left(\nu_{\omega}+1\right)>0$ and let

$$
V_{K, \omega}^{*}(x):=(\sup \{\varphi(x) / \varphi \in P S H(X, \omega), \varphi \leq 0 \text { on } K\})^{*}
$$

denote the Siciak extremal function of $K$ (see Section 5.1 in [GZ1]). Then

$$
\operatorname{Vol}_{\omega}(K) \leq \int_{X} \exp \left(-\gamma_{\omega} V_{K, \omega}^{*}\right) \omega^{n} \leq C_{2} T_{\omega}(K)^{\gamma_{\omega}},
$$

where $T_{\omega}(K):=\exp \left(-\sup _{X} V_{K, \omega}^{*}\right)$ denotes the Alexander capacity of $K$. It follows now from Theorem 7.1 in GZ1 that

$$
T_{\omega}(K) \leq e \exp \left[-\operatorname{Cap}_{\omega}(K)^{-1 / n}\right]
$$

which yields (4).

It follows therefore from Theorem 2.1 that there exists a unique continuous function $\varphi \in P S H(X, \omega)$ such that

$$
\mu=f \omega^{n}=\left(\omega+d d^{c} \varphi\right)^{n}, \text { with } \sup _{X} \varphi=-1,
$$

when $0 \leq f \in L^{p}\left(\omega^{n}\right), p>1$, with $\int_{X} f \omega^{n}=1$.

Actually we will be interested in measures with $L^{p}$-density with respect to a positive definite volume form $d \lambda$, while the smooth measure $\omega^{n}$ may vanish along a divisor. This does not make much difference, as follows from Hölder's inequality:

Lemma 3.2. Let $V$ be an n-dimensional compact normal Kähler space and let $\Omega$ be a smooth Kähler form on $V$. Let $\pi: X \rightarrow V$ be a resolution, $\omega=\pi^{*} \Omega$, and let $d \lambda$ be a positive definite smooth volume form on $X$.

If $\mu=f_{1} d \lambda$, with $f_{1} \in L^{p}(X, d \lambda)$ for some $p>1$, then there exists $p^{\prime}>1$ such that $\mu=f \omega^{n}$ and $f \in L^{p^{\prime}}\left(X, \omega^{n}\right)$. 
Proof. Observe that $\omega^{n}=E d \lambda$ for some smooth density $E \geq 0$ which vanishes along the exceptional locus of $\pi$; thus

$$
\mu=f_{1} d \lambda=f \omega^{n}, \text { where } f=f_{1} / E .
$$

Fix local coordinates $\left(z^{i}\right)_{1 \leq i \leq n}$ on a polydisk $\mathbb{D} \subset X$ and a local embedding $F: V \rightarrow \mathbb{C}^{m}$. Note that $E$ is comparable to $\left|\frac{\partial F}{\partial z^{1}} \wedge \ldots \wedge \frac{\partial F}{\partial z^{1}}\right|^{2} \simeq \sum_{i=1}^{r}\left|f_{i}\right|^{2}, f_{i}$ being holomorphic on $\mathbb{D}$. Therefore $E \in L_{l o c}^{\infty}(\mathbb{D})$ and $E^{-\alpha} \in L_{l o c}^{1}(\mathbb{D}, d \lambda)$ for some $0<\alpha<1$.

Choose $0<\alpha^{\prime}<\alpha$ such that $\frac{1}{p}+\frac{1}{\alpha}=\frac{1}{\alpha^{\prime}}$. Then $f^{\alpha^{\prime}}=f_{1}^{\alpha^{\prime}} E^{-\alpha^{\prime}}$ is the product of a function in $L^{p / \alpha^{\prime}}(d \lambda)$ and a function in $L^{\alpha / \alpha^{\prime}}(d \lambda)$; hence it is in $L_{l o c}^{1}(\mathbb{D}, d \lambda)$ by Hölder's inequality. A second application of Hölder's inequality yields

$$
\int_{\mathbb{D}} f^{1+\varepsilon} \omega^{n}=\int_{\mathbb{D}} f^{\epsilon} f_{1} d \lambda \leq\left(\int_{\mathbb{D}} f^{\epsilon q} d \lambda\right)^{1 / q}\left(\int_{\mathbb{D}} f_{1}^{p} d \lambda\right)^{1 / p}<+\infty,
$$

where $q$ denotes the conjugate exponent to $p$. This shows that $f \in L^{p^{\prime}}\left(\omega^{n}\right)$ if $p^{\prime}=1+\varepsilon>1$ is chosen so small that $\varepsilon q<\alpha^{\prime}$.

\subsection{Hölder continuity.}

Proposition 3.3. Assume $\omega_{\varphi}^{n}=f \omega^{n}, \omega_{\psi}=g \omega^{n}$, where $\varphi, \psi \in \operatorname{PSH}(X, \omega)$ are bounded and $f, g \in L^{p}\left(\omega^{n}\right), p>1$. Then for all $0<\gamma<2 /(2+n q)$,

$$
\|\varphi-\psi\|_{L^{\infty}(X)} \leq C\|\varphi-\psi\|_{L^{2}\left(\omega^{n}\right)}^{\gamma},
$$

where $q=p /(p-1)$ denotes the conjugate exponent to $p$.

Proof. Fix $\varepsilon>0$ and $\alpha>0$ to be chosen later. It follows from (2) and Propositions $2.6,3.1$ that

$$
\|\varphi-\psi\|_{L^{\infty}(X)} \leq \varepsilon+C_{1}\left[\operatorname{Cap}_{\omega}(|\varphi-\psi|>\varepsilon)\right]^{\alpha / n} .
$$

Applying the refined version of Lemma 2.3 which involves the uniform bound on $\|\varphi\|_{L^{\infty}(X)},\|\psi\|_{L^{\infty}(X)}$ (see inequality (3)), we obtain

$$
\operatorname{Cap}_{\omega}(|\varphi-\psi|>\varepsilon) \leq \frac{C_{2}}{\varepsilon^{n+2 / q}} \int_{X}|\varphi-\psi|^{2 / q}(f+g) \omega^{n} .
$$

It follows thus from Hölder's inequality that

$$
\operatorname{Cap}_{\omega}(|\varphi-\psi|>\varepsilon) \leq \frac{C_{3}|| f+g \|_{L^{p}}}{\varepsilon^{n+2 / q}}\left[\|\varphi-\psi\|_{L^{2}\left(\omega^{n}\right)}\right]^{2 / q} .
$$

Choose now $\varepsilon:=\|\varphi-\psi\|_{L^{2}}^{\gamma}$ where $0<\gamma<2 /(2+n q)$. Then

$$
\operatorname{Cap}_{\omega}(|\varphi-\psi|>\varepsilon) \leq C_{4}\left[\|\varphi-\psi\|_{L^{2}}\right]^{2 / q-\gamma(n+2 / q)} .
$$

We infer

$$
\|\varphi-\psi\|_{L^{\infty}(X)} \leq\|\varphi-\psi\|_{L^{2}}^{\gamma}+C_{5}\|\varphi-\psi\|_{L^{2}}^{\gamma^{\prime}}, \quad \text { where } \gamma^{\prime}=\frac{\alpha}{n}[2 / q-\gamma(n+2 / q)] \text {. }
$$

We finally choose $\alpha>0$ so large that $\gamma \leq \gamma^{\prime}$ and adjust the value of the constant $C$ : this yields the desired estimate.

Being able to control the $L^{\infty}$-norm of $\varphi-\psi$ by its $L^{2}$-norm is a powerful tool. If for instance $\psi=\varphi_{j}, \varphi$ satisfy the assumptions of Proposition 3.3-with $\varphi_{j}$ being uniformly bounded - and $\varphi_{j} \rightarrow \varphi$ in $L^{1}$, then $\varphi_{j} \rightarrow \varphi$ in $L^{2}\left(\omega^{n}\right)$; hence $\left(\varphi_{j}\right)$ actually uniformly converges towards $\varphi$. This yields the continuity of the map

$$
f \in L^{p}\left(\omega^{n}\right) \mapsto \varphi \in \mathcal{C}^{0}(X),
$$


where $\varphi$ is the unique $\omega$-psh solution to $\left(\omega+d d^{c} \varphi\right)^{n}=f \omega^{n}, \sup _{X} \varphi=-1$. Thus Theorem A is proved.

We now give an application of this estimate, which requires the manifold $X$ to be a rational homogenous manifold, i.e. $X=G / P$ where $G$ is a complex semi-simple algebraic group and $P$ a parabolic subgroup 5

Theorem 3.4. Assume $X$ is a rational homogeneous manifold. If $\mu=f \omega^{n}$ is a probability measure with density $0 \leq f \in L^{p}\left(\omega^{n}\right), p>1$, then the unique solution $\varphi \in P S H(X, \omega) \in \mathcal{C}^{0}(X)$ to the normalized Monge-Ampère equation

$$
\left(\omega+d d^{c} \varphi\right)^{n}=\mu=f \omega^{n}, \quad \sup _{X} \varphi=-1,
$$

is Hölder continuous of exponent $\gamma>0$, for all $\gamma<2 /(2+n q)$, where $q=p /(p-1)$ is the conjugate exponent to $p$.

Proof. Let $K$ be the maximal compact subgroup of $G$. Then, $K$ acts transitively on $X$. Furthermore, we may assume w.l.o.g. that $\omega$ is fixed by $K$. One can regularize $\omega$-psh functions by averaging over the Haar measure of $K$. This is very similar to the way one regularizes psh functions in $\mathbb{C}^{n}$ by using convolutions with an approximation of the identity for the convolution product. We refer the reader to $[\mathrm{Hu}$ and the Appendix of $\mathrm{G}$ ] for more details.

Let $\varphi_{h}$ be the $\omega$-psh function which is the translate of $\varphi$ by an element of $K$ which is at distance $h$ from the identity. We use the notation $\varphi_{h}$ by analogy with the $\mathbb{C}^{n}$-situation, where $\varphi_{h}(x)=\varphi(x+h)$. Since $\varphi$ is bounded, it has gradient in $L^{2}$; hence

$$
\left\|\varphi_{h}-\varphi\right\|_{L^{2}} \leq C|h|
$$

by using Cauchy-Schwarz inequality in a local chart. We can thus apply Proposition 3.3 to obtain that

$$
\left\|\varphi_{h}-\varphi\right\|_{L^{\infty}} \leq C^{\prime}|h|^{\gamma}
$$

for all $\gamma<2 /(2+n q)$. Since $\varphi_{h}(x) \simeq \varphi(x+h)$ in a local chart, this precisely means that $\varphi$ is Hölder-continuous of exponent $\gamma$.

\subsection{Regularity on the smooth locus.}

Theorem 3.5. Let $X$ be a projective algebraic complex manifold, $\omega_{0}$ a smooth semipositive closed $(1,1)$-form that is positive outside a complex subvariety $S \subset X$, and fix $\Omega$ to be a Kähler form on $X$. Assume that $\omega_{o}^{n}=D \Omega^{n}$, where $D^{-\varepsilon}$ is in $L^{1}\left(\Omega^{n}\right)$, and that $\left[\omega_{0}\right],[\Omega] \in N S_{\mathbb{R}}(X)$.

Let $\sigma_{1}, \ldots, \sigma_{p}$ (resp. $\tau_{1}, \ldots, \tau_{q}$ ) be holomorphic sections of some line bundle $L$ (resp $\left.L^{\prime}\right)$ on $X$. Fix $k \in \mathbb{R}_{\geq 0}, l \in \mathbb{R}_{\geq 0}$ and $F \in \mathcal{C}^{\infty}(X, \mathbb{R})$. Assume that

$$
\int_{X} \frac{1}{\left|\tau_{1}\right|^{2 l}+\ldots+\left|\tau_{q}\right|^{2 l}} \Omega^{n}<\infty \text { and } \int_{X} \frac{\left|\sigma_{1}\right|^{2 k}+\ldots+\left|\sigma_{p}\right|^{2 k}}{\left|\tau_{1}\right|^{2 l}+\ldots+\left|\tau_{q}\right|^{2 l}} e^{F} \Omega^{n}=\int_{X} \Omega^{n} .
$$

Then the unique bounded function $\varphi \in P S H\left(X, \omega_{0}\right)$ such that

$$
\left(\omega_{0}+d d^{c} \varphi\right)^{n}=\frac{\left|\sigma_{1}\right|^{2 k}+\ldots+\left|\sigma_{p}\right|^{2 k}}{\left|\tau_{1}\right|^{2 l}+\ldots+\left|\tau_{q}\right|^{2 l}} e^{F} \Omega^{n} \quad \text { and } \sup _{X} \varphi=-1
$$

is smooth outside $B=S \cup \bigcap_{i}\left\{\sigma_{i}=0\right\} \cup \bigcap_{i}\left\{\tau_{i}=0\right\}$.

\footnotetext{
${ }^{5}$ In particular, the cohomology class of $\omega$ is Kähler and $\omega$ itself can be supposed to be Kähler without loss of generality.
} 
Remark 3.6. This result should be compared with [Y], Theorem 8. Yau's result is stronger in many respects (there is no projectivity/rationality assumption and it gives a more precise regularity theory); on the other hand the conditions on the poles of the L.H.S. are less optimal than here.

We expect the projectivity/rationality assumptions to be superfluous. We also expect that a finer regularity theory might be developed for singular KE metrics depending on a finer analysis of the klt singularities involved.

The rest of this subsection will be devoted to the proof of Theorem 3.5. For the reader's convenience, we will treat two special cases before tackling the general case 6

Preliminary considerations. Thanks to Lemma 3.2 - here we use that $D^{-\varepsilon} \in L^{1}$ and Theorem 2.1. for every $t \in[0,1]$ there is a unique continuous function $\varphi_{t} \in$ $P S H\left(X, \omega_{0}+t \Omega\right)$ such that

$$
\left(\omega_{o}+t \Omega+d d^{c} \varphi_{t}\right)^{n}=C_{t} \frac{\left|\sigma_{1}\right|^{2 k}+\ldots+\left|\sigma_{p}\right|^{2 k}}{\left|\tau_{1}\right|^{2 l}+\ldots+\left|\tau_{q}\right|^{2 l}} e^{F} \Omega^{n} \text { and } \sup _{X} \varphi_{t}=-1,
$$

where $C_{t}>0$ is an adequate normalization constant and $\left\|\varphi_{t}\right\|_{\mathcal{C}^{0}(X)}$ is uniformly bounded by a constant independant of $t \geq 0$.

We cannot use $[\mathrm{Y}]$, Theorem 8 , p. 403, right away to ensure that $\left(\varphi_{t}\right)$ is smooth outside $B$ for $t>0$, since our integral condition is stronger than his. However we can use $[\mathrm{Y}]$, Theorem 3, p. 365, to conclude that, in case $\bigcap_{i}\left\{\tau_{i}=0\right\}=\emptyset,\left(\varphi_{t}\right)$ is smooth outside $B$ and $d d^{c} \varphi_{t}$ is a form whose coefficients are globally bounded on $X$. Since this does not imply ellipticity if $\bigcap_{i}\left\{\sigma_{i}=0\right\} \neq \emptyset$, this does not imply higher regularity on the whole of $X$.

The required uniformity in $t>0$ is not proved in $[\mathrm{Y}]$. To deal with this case, we use a nice trick due to $\mathrm{H}$. Tsuji $\mathrm{Ts}$.

The simplest case. First, assume $\bigcap_{i}\left\{\sigma_{i}=0\right\} \cup \bigcap_{i}\left\{\tau_{i}=0\right\}=\emptyset$. Hence the family of equations under consideration can be rewritten as

$$
\left(\omega_{o}+t \Omega+d d^{c} \varphi_{t}\right)^{n}=C_{t} e^{F} \Omega^{n}
$$

$F$ being smooth.

Tsuji's trick is as follows. By Kodaira's lemma, there exists $E$, an effective Cartier divisor of $X$, such that $\left[\omega_{o}\right]=\left[\kappa_{\varepsilon}\right]+\varepsilon[E]$ where $\left[\kappa_{\varepsilon}\right]$ is ample; hence we may choose a representative $\kappa_{\varepsilon}$ which is a Kähler form for every $\varepsilon>0$ small enough. We may actually assume $E$ contains $B$ and use a family of $E$ such that $\bigcap \operatorname{Supp}(E)=B$, by Nakamaye's theorem on base loci [Na].

Actually, despite the notation, it will NOT be necessary to let $\varepsilon$ decrease to 07 and we will fix once for all such an $\varepsilon>0$.

Let $\sigma \in H^{0}\left(X, \mathcal{O}_{X}(E)\right)$ be the canonical section vanishing on $E$ with the appropriate multiplicity. We can fix a smooth hermitian metric on this line bundle such that the Poincaré Lelong equation holds:

$$
\omega_{o}=\kappa_{\varepsilon}+\varepsilon[E]-\varepsilon d d^{c} \log |\sigma|^{2} .
$$

\footnotetext{
${ }^{6}$ Notice that apart from the $\mathcal{C}^{0}$-estimate with degenerate L.H.S., the methods used here are standard and are in $[\mathrm{Y}],[\mathrm{Ts}]$ and $[\mathrm{Kö}]$. Higher regularity in [TZ] is treated along similar lines given the $L^{\infty}$-estimate the authors announce.

${ }^{7}$ This technical device could be useful to study finer regularity results.
} 
The function $\phi_{t}:=\varphi_{t}-\varepsilon \log |\sigma|^{2}$ is smooth in $X \backslash E$ and is a classical solution to the PDE

$$
\left(\kappa_{\varepsilon}+t \Omega+d d^{c} \phi_{t}\right)^{n}=e^{F_{\varepsilon, t}}\left(\kappa_{\varepsilon}+t \Omega\right)^{n},
$$

where $\left(F_{\varepsilon, t}\right)_{1>t>0}$ is uniformly bounded in the $\mathcal{C}^{\infty}(X)$-topology of functions and $\kappa_{t}=\kappa_{\varepsilon}+t \Omega$ is uniformly bounded in the $\mathcal{C}^{\infty}$-topology of Kähler forms on $X$.

We can use the result of the calculation in $Y$, Section 2. The important formula is (2.22), p. 351, and in a subsidiary fashion (2.21). In these formulae, at each point $p \in X-E$, an adequate system of normal coordinates for $\kappa_{t}$ is constructed and comparing the notation here and there, we substitute $n$ for $m, \kappa_{t}$ for $g_{i \bar{j}}, \kappa_{t}+d d^{c} \phi_{t}$ for $g_{i \bar{j}}^{\prime}, \phi_{t}$ for $\phi$ and $F_{\varepsilon, t}$ for $F$. The operator $\Delta$ is the Laplace operator (with the analyst's sign) of $\kappa_{t}$ and $\Delta^{\prime}$ the Laplace operator of $\kappa_{t}+d d^{c} \phi_{t}$. Also $R_{i \bar{i} i \bar{l}}=R_{i \bar{i} l \bar{l}}^{t}$ is the holomorphic bissectional curvature of $\kappa_{t}$ expressed in the above system of normal coordinates.

Since $\kappa_{t}$ is uniformly bounded in the $\mathcal{C}^{2}$-topology of Kähler forms, then certainly there are constants $C=C_{\varepsilon}$ independent of $t$ such that (2.21) holds and $C^{\prime}$ also independent of $t$ such that $C^{\prime}>\inf R_{i \bar{i} i \bar{l}}^{t}$.

After these substitutions are made, (2.22), p. 351, reads:

$e^{C \phi_{t}} \Delta^{\prime}\left(e^{-C \phi_{t}}\left(n+\Delta \phi_{t}\right)\right) \geq \Delta\left(F_{\varepsilon, t}\right)-n^{2} C^{\prime}-C n\left(n+\Delta \phi_{t}\right)+e^{-\frac{F_{\varepsilon, t}}{n-1}}\left(n+\Delta \phi_{t}\right)^{\frac{n}{n-1}}$.

We can fix constants $C_{i}$ independent of $t$ such that

$$
\Delta F_{\varepsilon, t} \geq C_{1} \quad \text { and } \quad e^{-F_{\varepsilon, t} / n-1} \geq C_{3}>0 .
$$

Thus setting $y=n+\Delta \phi_{t}$ yields

$$
e^{C \phi_{t}} \Delta^{\prime}\left(e^{-C \phi_{t}}\left(n+\Delta \phi_{t}\right)\right) \geq C_{5}+C_{6} y+e^{C_{7}} y^{\frac{n}{n-1}} .
$$

Now by definition

$$
e^{-C \phi_{t}}\left(n+\Delta \phi_{t}\right)=|\sigma|^{+C \varepsilon} e^{-C \varphi_{t}}\left(n+\Delta \varphi_{t}+\varepsilon \Delta \log |\sigma|^{2}\right) .
$$

For each $t>0$ the functions $\varphi_{t}, \varepsilon \Delta \log |\sigma|^{2}$ and $\Delta \varphi_{t}$ are bounded on $X$. Hence the positive function $e^{-C \phi_{t}}\left(n+\Delta \phi_{t}\right)$ is continuous on $X$, vanishes on $E$ and is smooth on $X-E$. Its maximum is achieved at some point $p_{t} \notin E$. It follows from the maximum principle that

$$
0 \geq C_{5}+C_{6} y+e^{C_{7}} y^{\frac{n}{n-1}} \text { at point } y=y\left(p_{t}\right) .
$$

Therefore $y \leq C_{8}$ with a constant independent of $t>0$. Now $e^{-C \phi_{t}\left(p_{t}\right)}=$ $\left|\sigma\left(p_{t}\right)\right|^{+C \varepsilon} e^{-C \varphi_{t}\left(p_{t}\right)}$. Using the uniform $\mathcal{C}^{0}$-estimate for $\varphi_{t}$, we get $0 \leq\left(n+\Delta \phi_{t}\right) \leq$ $C_{9} e^{+C \phi_{t}}$. Since $\left|\varphi_{t}\right|$ and $\varepsilon \Delta \log |\sigma|^{2}$ are uniformly bounded by a constant independent of $t>0$, we infer

$$
\left(n+\Delta \varphi_{t}\right) \leq C_{10}|\sigma|^{-C \varepsilon}=C_{10}|\sigma|^{-C_{\varepsilon} \varepsilon} .
$$

This yields a $t$-independent $\mathcal{C}^{0}$ - estimate of $d d^{c} \varphi_{t}$ on the compact subsets of $X-E 8$

Standard arguments of the theory of complex Monge-Ampère equations give an interior estimate of $\varphi_{t}$ in $\mathcal{C}_{\text {loc }}^{k, \alpha}(X-E)$ for every $\left.k \geq 2, \alpha \in\right] 0,1$ [ which is independent of $t>0$ (see for instance Theorem 5.1, p. 15, in [B2]). Hence the family $\left(\varphi_{t}\right)_{t>0}$ is precompact in every $\mathcal{C}_{l o c}^{k, \alpha}(X-E)$. Its cluster values are cluster values in $\mathcal{C}^{0}(X-E)$; hence they are all equal to $\left.\varphi\right|_{X-E}$. This implies $\varphi \in \mathcal{C}_{l o c}^{k, \alpha}(X-E)$, hence that $\varphi \in \mathcal{C}^{\infty}(X-E)$.

\footnotetext{
${ }^{8}$ Note that $C=C_{\varepsilon}$ and that $\varepsilon C_{\varepsilon}$ might blow up as $\varepsilon$ goes to 0 .
} 
Case where $\bigcap_{i}\left\{\tau_{i}=0\right\}=\emptyset 9$ We study here the equation

$$
\left(\omega_{o}+t \Omega+d d^{c} \varphi_{t}\right)^{n}=C_{t}\left(\left|\sigma_{1}\right|^{2 k}+\ldots+\left|\sigma_{p}\right|^{2 k}\right) e^{F} \Omega^{n} .
$$

The first few steps of the preceding argument can be repeated without changes. Next we apply formula (2.22) in $[\mathrm{Y}]$ as earlier, except that we set $F=F_{\varepsilon, t}+$ $\log \|s\|^{(2 k)}$, where $\|s\|^{(2 k)}:=\left|\sigma_{1}\right|^{2 k}+\ldots+\left|\sigma_{p}\right|^{2 k}$. This yields

$$
\begin{aligned}
e^{C \phi_{t}} \Delta^{\prime}\left(e^{-C \phi_{t}}\left(n+\Delta \phi_{t}\right)\right) \geq & \Delta F_{\varepsilon, t}+\Delta \log \|s\|^{(2 k)}-n^{2} C^{\prime}-C n\left(n+\Delta \phi_{t}\right) \\
& +\left(\frac{e^{-F_{\varepsilon, t}}}{\|s\|^{(2 k)}}\right)^{1 /(n-1)}\left(n+\Delta \phi_{t}\right)^{\frac{n}{n-1}}
\end{aligned}
$$

We recall the two preceding inequalities and observe two new ones that are available:

$$
\begin{aligned}
\Delta F_{\varepsilon, t} \geq C_{1} \quad & \text { and } \quad e^{-F_{\varepsilon, t} / n-1} \geq C_{3}>0 ; \\
\Delta \log \|s\|^{(2 k)} \geq C_{2} \quad & \text { and } \quad C_{4} \geq\|s\|^{(2 k)} .
\end{aligned}
$$

Setting as earlier $y=n+\Delta \phi_{t}$, we get

$$
e^{C \phi_{t}} \Delta^{\prime}\left(e^{-C \phi_{t}}\left(n+\Delta \phi_{t}\right)\right) \geq C_{5}+C_{6} y+e^{C_{7}} y^{\frac{n}{n-1}} .
$$

After this point, the proof is entirely the same as before.

Remark 3.7. In order to carry out the second-order a priori estimate, one needs information that only depend $\operatorname{on}_{\sup _{X}} F$ and $\inf _{X} \Delta F$. This is pointed out in [Y], p. 351, and it is the basis for the proof of [Y], Theorem 3.

3.4. Formal reduction of the general case to the second case using smooth orbifolds. In order to carry out the present argument, which in essence is just a change of variables $z \rightsquigarrow \zeta=z^{1 / m}$, we need to use analysis on certain smooth orbifolds. We will not give complete definitions since they are in the recent reference BGK, Section 2, pp. 560-564; see also $\mathrm{MO}$ and the references therein.

Let $(X, \Delta)$ be a smooth orbifold pair. By this we mean that we have the prime decomposition $\Delta=\sum_{i}\left(1-m_{i}^{-1}\right) E_{i}$ where $m_{i} \in \mathbb{N}^{*}$ is an integer. We assume that $\operatorname{supp}(\Delta)$ is a simple normal crossing divisor. Then, a classical construction surveyed in $\mathrm{BGK}$ enables one to construct an orbifold $[X, \Delta]$ with a 1-morphism of orbifolds $c:[X, \Delta] \rightarrow X$ with the following properties:

- $c$ is the reduction to the coarse moduli space of $[X, \Delta]$.

- $c_{X-\operatorname{Supp}(\Delta)}: U:=[X, \Delta] \times_{X}(X-\operatorname{Supp}(\Delta)) \rightarrow X-\operatorname{Supp}(\Delta)$ is an isomorphism. Hence $U$ is an open suborbifold of $[X, \Delta]$ which is an old-fashioned manifold).

- For every open polydisk $\mathbb{D} \subset X$ with local coordinates $z_{1}, \ldots, z_{n}$ such that $\operatorname{Supp}(\Delta)=\left\{\prod_{j=1}^{p} z_{j}=0\right\}[X, \Delta] \times_{X} \mathbb{D}=\left[\mathbb{D}^{\prime} / G_{l o c}\right]$.

In this formula, the local isotropy group is $G_{l o c}=\prod_{j=1}^{p} \mathbb{Z} / m_{j} \mathbb{Z}, m_{j}$ is the integer multiplicity of the divisor $E_{i_{j}}$ such that $E_{i_{j}} \cap \mathbb{D}=\left\{z_{j}=0\right\}, G_{l o c}$ acts on the polydisk $\mathbb{D}^{\prime}$ by $\left(\zeta_{1}, \ldots, \zeta_{p}\right) \cdot\left(z_{1}^{\prime}, \ldots, z_{n}^{\prime}\right)=\left(\zeta_{1} z_{1}^{\prime}, \ldots, \zeta_{p} z_{p}^{\prime}, z_{p+1}^{\prime}, \ldots\right) 10$

The orbifold 1-morphism $\left[\mathbb{D}^{\prime} / G_{l o c}\right] \rightarrow \mathbb{D}$ is induced by $\varkappa_{l o c}:\left(z_{1}^{\prime}, . ., z_{n}^{\prime}\right) \mapsto$ $\left(\left(z_{1}^{\prime}\right)^{m_{1}}, \ldots\right)$.

- For sufficiently divisible $s, c_{*} \mathcal{O}_{[X, \Delta]}\left(s K_{[X, \Delta]}\right)=\mathcal{O}_{X}\left(s\left(K_{X}+\Delta\right)\right)$.

\footnotetext{
${ }^{9}$ It suffices to consider this case for constructing singular KE metrics on algebraic varieties with canonical singularities

${ }^{10}$ The usual isomorphism of $\mathbb{Z} / m \mathbb{Z}$ with the group of $m$-th root of unity is used.
} 
It is possible to define all the basic concepts of Kähler geometry on orbifolds such as smooth functions, Kähler metrics, etc. The principle is to think of $\varkappa_{l o c}^{-1}$ as a (multivalued) smooth coordinate chart.

A continuous function on $[X, \Delta]$ is a continuous function on $X$. A Radon measure on $[X, \Delta]$ is a Radon measure on $X$.

A smooth function $f$ on $[X, \Delta]$ is a continuous function on $X$ such that for every local chart $\varkappa_{l o c}^{*} f$ is smooth. In particular $f$ is Hölder continuous.

A Kähler metric $\Omega_{[X, \Delta]}$ on $[X, \Delta]$ is a Kähler metric $\Omega_{X-S u p p(\Delta)}$ on $X-\operatorname{Supp}(\Delta)$ with the property that $\varkappa_{l o c}^{*} \Omega$ extends to a smooth Kähler metric on $\mathbb{D}^{\prime}$. In particular, it also extends as a closed Kähler current on $X$ with Hölder potentials.

The pull back of a Kähler form on $X$ to $[X, \Delta]$ is a semi-positive closed $(1,1)$-form that is actually cohomologous to a Kähler clas: 11 .

Observe that $\varkappa_{l o c}^{*} d z_{l}=m_{i_{l}}\left(z_{l}^{\prime}\right)^{m_{i_{l}}-1} d z_{l}^{\prime}$; hence a smooth volume form on $[X, \Delta]$ can be interpreted as a volume form $v$ on $X-\operatorname{Supp}(\Delta)$ such that

$$
v \text { is comparable to } \frac{\prod_{l=1}^{n}\left(\sqrt{-1} d z_{l} \wedge d \bar{z}_{l}\right)}{\prod_{l=1}^{p}\left|z_{l}\right|^{2\left(1-1 / m_{i_{l}}\right)}} .
$$

In case the pair $\left(X, l^{-1}\left(\tau_{1}\right)\right)$ is an orbifold pair, the equation

$$
\left(\omega_{o}+d d^{c} \varphi_{t}\right)^{n}=C_{t} \frac{\left|\sigma_{1}\right|^{2 k}+\ldots+\left|\sigma_{p}\right|^{2 k}}{\left|\tau_{1}\right|^{2 l}} e^{F} \Omega_{X}^{n}
$$

can be interpreted on $[X, \Delta]$ as an equation of the form

$$
\left(c^{*} \omega_{o}+d d^{c} \varphi_{t}\right)^{n}=C_{t}\left(\left|\sigma_{1}\right|^{2 k}+\ldots+\left|\sigma_{p}\right|^{2 k}\right) e^{F} \Omega_{[X, \Delta]}^{n} .
$$

The method used to analyze the case where $\bigcap_{i}\left\{\tau_{i}=0\right\}=\emptyset$ extends with almost no changes to the orbifold case. Hence the unique continuous solution of equation (5) is smooth outside its singular locus if $\left(X, \frac{1}{l}\left(\tau_{1}\right)\right)$ is an orbifold pair.

Under the more general hypothesis that $\int_{X}\left|\tau_{1}\right|^{-2 l}<\infty$ and $\frac{1}{l}\left(\tau_{1}\right)$ is a divisor with simple normal crossings, then we can construct an orbifold pair $(X, \Delta)$ with $0 \leq \frac{1}{l}\left(\tau_{1}\right) \leq \Delta$ and we are back to the previous case.

For the most general case, consider the ideal $\mathcal{I}$ generated by the $t_{i}$ and fix $\mu: X^{\prime} \rightarrow X$ a log-resolution of $(X, \mathcal{I})$. Then we are back to the previous case, with an equation on $X^{\prime}$. This ends the proof of Theorem 3.5.

In certain rare circumstances, there is a finite smooth covering $Y \rightarrow X$ such that $Y / G=X$ and $[Y / G]=[X, \Delta]$ and the argument we use here reduces to a $G$-equivariant argument on $Y$.

Remarks 3.8. If we start with $\omega_{o}$ Kähler and the log-resolution is non-trivial, $\mu^{*} \omega_{o}$ is not Kähler anymore.

This method that dates back to [Kö] can be used to prove a variant of [Y], Theorem 7, p. 399, where the divisor of $\sigma_{2}$ is a simple normal crossing divisor, under the sole assumption that $\int_{M}\left|\sigma_{2}\right|^{-2 k_{2}}<\infty$.

Now, it could not have been used to prove Theorem 8, p. 403, in 1978 since log-resolutions force the use of Monge-Ampère equations with degenerate L.H.S., for which the $\mathcal{C}^{0}$-estimate proved here was not available then.

\footnotetext{
${ }^{11}$ Here no reference can be given. But it is easy to extend the gluing methods for Kähler forms developed in $[\mathrm{Dem} 3]$ and $[\mathrm{Pa}$ to orbifolds. Hence $[\mathrm{DP}]$ extends to Kähler orbifolds.
} 


\section{More Monge-Ampère EQuations}

As we aim at constructing singular Kähler-Einstein metrics, it is important to consider Monge-Ampère equations of the type

$$
\left(\omega+d d^{c} \varphi\right)^{n}=e^{t \varphi} \mu,
$$

where $\mu$ is a probability measure which satisfies condition $\mathcal{H}(\alpha, A, \omega)$ (see Definition 1.3 ), and $t$ is a real parameter. The case $t=0$, treated in Theorem 2.1, will correspond to Ricci-flat metrics (see Section 6). We focus here on the case $t>0$.

Theorem 4.1. Let $\mu$ be a probability measure which satisfies condition $\mathcal{H}(\alpha, A, \omega)$ and fix $t>0$. There exists a unique function $\varphi_{t} \in P S H(X, \omega) \cap L^{\infty}(X)$ such that

$$
\left(\omega+d d^{c} \varphi_{t}\right)^{n}=e^{t \varphi_{t}} \mu .
$$

Furthermore $\varphi_{t}$ is continuous provided $(X,[\omega])$ enjoys the continuous approximation property.

Proof. The uniqueness easily follows from the comparison principle as we explain in Proposition 4.3 below. We are going to prove the existence by a fixed point method.

Fix $\psi \in \mathcal{E}^{1}(X, \omega)$ such that $\int_{X} \psi d \mu=0$, and let us consider the equation

$$
\left(\omega+d d^{c} \varphi\right)^{n}=e^{t \psi-c_{\psi}} \mu,
$$

where the constant $c_{\psi}:=\log \left[\int_{X} e^{t \psi} d \mu\right]$ is chosen so that

$$
1=\int_{X}\left(\omega+d d^{c} \varphi\right)^{n}=e^{-c_{\psi}} \int_{X} e^{t \psi} d \mu .
$$

Observe that $\mu_{\psi}:=e^{t \psi-c_{\psi}} \mu$ satisfies condition $\mathcal{H}\left(\alpha, A_{\psi}, \omega\right)$, where $A_{\psi}=$ $\exp \left(t \sup _{X} \psi-c_{\psi}\right)$. It follows therefore from Theorem 2.1 that there exists a unique bounded function $\varphi \in P S H(X, \omega)$ which is a solution to $(M A(\psi))$ and normalized by $\int_{X} \varphi d \mu=0$. We use here this linear normalization rather than the non-linear sup-normalization: they are comparable thanks to Proposition 2.7 in [GZ1], which shows that

$$
-M_{\mu} \leq \int_{X} u d \mu-\sup _{X} u \leq 0,
$$

for all functions $u \in P S H(X, \omega)$ and for some uniform constant $M_{\mu}>0$. Since $\int_{X} \psi d \mu=0$, we infer

$$
0 \leq \mathcal{E}_{\omega}(\varphi):=\int_{X}|\varphi| \omega_{\varphi}^{n}=e^{-c_{\psi}} \int_{X}|\varphi| e^{t \psi} d \mu \leq 2 M_{\mu} e^{t M_{\mu}},
$$

by observing that $c_{\psi} \geq 0$ since $t \geq 0$, and

$$
\int_{X}|\varphi| d \mu \leq \int_{X}\left|\varphi-\sup _{X} \varphi\right| d \mu+\sup _{X} \varphi \leq 2 M_{\mu},
$$

since $\int_{X} \varphi d \mu=0$.

The important fact here is that the energy $\mathcal{E}_{\omega}(\varphi)$ of $\varphi$ is bounded from above by a constant $M_{0}:=2 M_{\mu} e^{t M_{\mu}}$ which is independent of $\psi$. We have thus defined an operator

$$
T: \psi \in \mathcal{C}_{M} \mapsto \varphi \in \mathcal{C}_{M_{0}}
$$


which associates to $\psi \in \mathcal{C}_{M}$ the unique solution $\varphi \in \mathcal{C}_{M_{0}}$ to $(M A(\psi))$, where

$$
\mathcal{C}_{M}:=\left\{\psi \in \mathcal{E}^{1}(X, \omega) / \int_{X} \psi d \mu=0 \text { and } \mathcal{E}_{\omega}(\psi) \leq M\right\} .
$$

It follows from GZ2 that $\mathcal{E}^{1}(X, \omega)$ is convex. So is the subset of functions $\psi \in$ $\mathcal{E}^{1}(X, \omega)$ such that $\int_{X} \psi d \mu=0$. The set $\mathcal{C}_{M}$ is not convex, but it is relatively compact in $L^{1}(X)$ and its closed convex hull $\hat{\mathcal{C}}_{M}$ is contained in $\mathcal{C}_{\kappa_{n} M}$ for some uniform constant $\kappa_{n}$ which only depends on the dimension of $X$ : this follows from easy computations (see Proposition 2.10 in GZ2]). Therefore $T$ maps the compact convex set $\hat{\mathcal{C}}_{M}$ into itself if $M$ is large enough.

We claim that $T$ is continuous. Let $\left(\psi_{j}\right) \in \mathcal{C}_{M}^{\mathbb{N}}$ be a sequence of functions which converges in $L^{1}(X)$ towards $\psi \in \mathcal{C}_{M}$. We need to show that $\varphi_{j}:=T\left(\psi_{j}\right)$ converges in $L^{1}(X)$ towards $T(\psi)$. Since the set $\left\{u \in P S H(X, \omega) / \int_{X} u d \mu=0\right\}$ is relatively compact in $L^{1}(X)$ (see Proposition 2.7 in [GZ1]), we can assumerelabelling if necessary - that $\left(\varphi_{j}\right)$ converges in $L^{1}(X)$ towards a function $\varphi \in$ $P S H(X, \omega)$. We show in Lemma 4.2 below that $\left(\varphi_{j}\right)$ converges in $L^{1}(\mu)$ towards $\varphi$. In particular $\int_{X} \varphi d \mu=0$ and, passing to a subsequence if necessary, we can assume that $e^{t \psi_{j}(x)} \rightarrow e^{t \psi(x)}$ for $\mu$ almost every point $x$. Set

$$
\hat{\varphi}_{j}:=\left(\sup _{l \geq j} \varphi_{l}\right)^{*} \text { and } \check{\psi}_{j}:=\inf _{l \geq j} \psi_{l} .
$$

Observe that $\left(\hat{\varphi}_{j}\right)$ decreases towards $\varphi$, while $\left(e^{t \check{\psi}_{j}}\right)$ increases towards $e^{t \psi}$ at $\mu$ almost every point. The energy of $\hat{\varphi}_{j}$ is controlled by that of $\varphi_{j}$ since $\hat{\varphi}_{j} \geq \varphi_{j}$ (see Lemma 2.3 in GZ2]), and $\mathcal{E}_{\omega}\left(\varphi_{j}\right) \leq M_{0}$ by $(5)$; therefore $\varphi \in \mathcal{E}^{1}(X, \omega)$ and $\left(\omega+d d^{c} \hat{\varphi}_{j}\right)^{n} \rightarrow\left(\omega+d d^{c} \varphi\right)^{n}$. It follows from an inequality of J. -P. Demailly Dem1] that

$$
\left(\omega+d d^{c} \hat{\varphi}_{j}\right)^{n} \geq e^{t \check{\psi}_{j}-\hat{c}_{j}} \mu
$$

where $\hat{c}_{j}:=\sup _{l \geq j} c_{\psi_{l}}$. Observe that $\hat{c}_{j} \rightarrow c_{\psi}$; thus

$$
\left(\omega+d d^{c} \varphi\right)^{n} \geq e^{t \psi-c_{\psi}} \mu .
$$

Since these are two probability measures, there is actually equality; hence $\varphi=T(\psi)$ : this shows that $T$ is continuous.

We can now invoke the Schauder fixed point theorem, which yields a fixed point $\varphi=T(\varphi), \varphi \in \mathcal{C}_{M}$. The function $\varphi$ is automatically bounded (by Theorem 2.1, since $e^{t \varphi-c_{\varphi}} \mu$ satisfies $\mathcal{H}\left(\alpha, A^{\prime}, \omega\right)$ ); hence $\Phi:=\varphi-t^{-1} c_{\varphi}$ is the solution we were looking for.

Lemma 4.2. The functions $\varphi_{j}=T\left(\psi_{j}\right)$ (respectively $e^{t \psi_{j}}$ ) converge in $L^{1}(\mu)$ towards $\varphi$ (respectively $\left.e^{t \psi}\right)$.

Proof. We first show that $\left(\varphi_{j}\right)$ converges to $\varphi$ in $L^{1}(\mu)$. Observe that the sequence $\left(\varphi_{j}\right)$ is uniformly bounded: this follows from Theorem 2.1 since $\left(\omega+d d^{c} \varphi_{j}\right)^{n}$ satisfies $\mathcal{H}\left(\alpha, A_{j}, \omega\right)$, where $A_{j}=e^{t \sup _{X} \psi_{j}-c_{\psi_{j}}} A \leq e^{t M_{\mu}} A$ is bounded from above. It follows then from standard arguments that $\int_{X} \varphi_{j} d \mu \rightarrow \int_{X} \varphi d \mu$ (see e.g. the proof of Lemma 5.2 in $\mathrm{Ce}$ ).

Fix $\varepsilon>0$ and let $G$ be an open set of $X$ such that $\varphi$ is continuous on $X \backslash G$ and $\operatorname{Cap}_{\omega}(G) \leq \varepsilon$ (see Corollary 3.8 in [GZ1]). By Hartogs' lemma, $\varphi_{j} \leq \varphi+\varepsilon$ on the 
compact set $X \backslash G$, if $j \geq j_{\varepsilon}$. Observe that

$$
\int_{X \backslash G}\left|\varphi-\varphi_{j}\right| d \mu \leq 2 \varepsilon+\int_{X \backslash G}\left[\varphi-\varphi_{j}\right] d \mu \leq 3 \varepsilon,
$$

if $j \geq j_{\varepsilon}^{\prime}$. On the other hand since $\mu$ satisfies $\mathcal{H}(\alpha, A, \omega)$, we get

$$
\int_{G}\left|\varphi-\varphi_{j}\right| d \mu \leq 2 M \mu(G) \leq 2 M A \varepsilon^{1+\alpha},
$$

where $M=\sup _{j}\left\|\varphi_{j}\right\|_{L^{\infty}(X)}$. This shows that $\left\|\varphi-\varphi_{j}\right\|_{L^{1}(\mu)} \rightarrow 0$.

The proof for $\left(e^{t \psi_{j}}\right)$ is similar: it suffices to note that the functions $u_{j}:=$ $e^{t \psi_{j}-t \sup _{X} \psi_{j}}$ are $\omega$-psh and uniformly bounded. One can then apply the rest of the argument.

Proposition 4.3. Let $\varphi, \psi \in \mathcal{E}^{1}(X, \omega)$ and $t>0$ be such that

$$
\left(\omega+d d^{c} \varphi\right)^{n} e^{-t \varphi}=\left(\omega+d d^{c} \psi\right)^{n} e^{-t \psi} .
$$

Then $\varphi \equiv \psi$.

Proof. It follows from the comparison principle (see [GZ2]) that

$$
\int_{(\varphi<\psi)}\left(\omega+d d^{c} \psi\right)^{n} \leq \int_{(\varphi<\psi)}\left(\omega+d d^{c} \varphi\right)^{n}=\int_{(\varphi<\psi)} e^{t(\varphi-\psi)}\left(\omega+d d^{c} \psi\right)^{n} .
$$

Since $e^{t(\varphi-\psi)}<1$ on $(\varphi<\psi)$, we infer $\varphi \geq \psi$ for $\nu$ almost every point, where $\nu=\left(\omega+d d^{c} \psi\right)^{n}$. Reversing the roles of $\varphi, \psi$ yields $\varphi=\psi$ for $\nu$ almost every point. Therefore $\left(\omega+d d^{c} \varphi\right)^{n}=\left(\omega+d d^{c} \psi\right)^{n}$; hence $\varphi-\psi=c$ is constant by Theorem 3.4 in GZ2. Finally $c=0$ since $e^{t c}=1$ and $t>0$.

When $K_{X}$ is nef and big, H. Tsuji constructed in [Ts] - using Kähler-Ricci flow techniques - a function $\psi \in P S H(X, \omega)$ such that

$$
\int_{X} e^{t \psi} d \mu=1, \psi \in \mathcal{C}^{\infty}(X \backslash E) \text { and }\left(\omega+d d^{c} \psi\right)^{n}=e^{t \psi} \mu \text { in } X \backslash E,
$$

where $E$ is the exceptional locus of the map associated to the base point free linear system $\left|N K_{X}\right|, N \in \mathbb{N}$ big enough and the current $T_{K E}=\omega+d d^{c} \psi$ defines a Kähler-Einstein metric. This function coincides with our solution thanks to the following unicity result.

Proposition 4.4. Let $\mu$ be a probability measure and $t>0$. Let $\varphi, \psi \in P S H(X, \omega)$ be such that $\int_{X} e^{t \varphi} d \mu=\int_{X} e^{t \psi} d \mu=1$. Assume $\varphi \in \mathcal{E}^{1}(X, \omega)$ is a global solution to the complex Monge-Ampère equation $\left(\omega+d d^{c} \varphi\right)^{n}=e^{t \varphi} \mu$, while $\psi \in \mathcal{C}^{0}(X \backslash E)$ satisfies $\left(\omega+d d^{c} \psi\right)^{n}=e^{t \psi} \mu$ only in $X \backslash E$.

Then $\psi \in \mathcal{E}^{1}(X, \omega)$ and $\psi \equiv \varphi$.

Proof. Set $\psi_{j}:=\max (\psi,-j) \in P S H(X, \omega) \cap L^{\infty}(X)$. Observe that the probability measures $\left(\omega+d d^{c} \psi_{j}\right)^{n}$ converge in $X \backslash E$ towards the measure $\nu=e^{t \psi} \mu$. Since $\nu(X)=\nu(X \backslash E)=1$, it follows that $\left(\omega+d d^{c} \psi_{j}\right)^{n}$ converges to $\nu$ on all of $X$.

Fix $\varepsilon>0$ and set $v_{\varepsilon}:=(\psi+\varepsilon v) /(1+\varepsilon) \in P S H(X, \omega)$, where $v \in P S H(X, \omega)$, $v \leq 0$, is such that $e^{v}$ is continuous and $(v=-\infty)=E$. It follows from Lemma 2.3 that for all $s>0$,

$$
\operatorname{Cap}_{\omega}\left(\psi_{j}<-s-1\right) \leq \int_{\left(\psi_{j}<-s\right)}\left(\omega+d d^{c} \psi_{j}\right)^{n} \leq \int_{\left(v_{\varepsilon} \leq-s /(1+\varepsilon)\right)}\left(\omega+d d^{c} \psi_{j}\right)^{n} .
$$


Observe that $e^{v_{\varepsilon}}$ is continuous on $X$; hence the sublevel sets $\left(v_{\varepsilon} \leq c\right)$ are compact. We infer, letting $j \rightarrow+\infty$,

$$
\operatorname{Cap}_{\omega}(\psi<-s-1) \leq \int_{\left(v_{\varepsilon} \leq-s /(1+\varepsilon)\right)} e^{t \psi} d \mu .
$$

Letting $\varepsilon$ go to zero and using that $\mu(X)=1$ yields

$$
\operatorname{Cap}_{\omega}(\psi<-s-1) \leq \int_{(\psi<-s)} e^{t \psi} d \mu \leq e^{-s} .
$$

Therefore the capacity of the sublevel sets of $\psi$ decreases fast as $s \rightarrow+\infty$; hence by Lemma 5.1 in GZ2 we get $\psi \in \mathcal{E}^{1}(X, \omega)$. Since $e^{-t \varphi}\left(\omega+d d^{c} \varphi\right)^{n} \equiv e^{-t \psi}\left(\omega+d d^{c} \psi\right)^{n}$, it follows from Proposition 4.3 that $\varphi \equiv \psi$.

Theorem 4.5. Let $X$ be a projective algebraic complex manifold, $\omega_{0}$ a smooth semi-positive closed $(1,1)$-form that is Kähler outside a complex subvariety $S \subset X$, and fix $\Omega$ to be a Kähler form on $X$. Assume that $\omega_{o}^{n}=D \Omega^{n}$, where $D^{-\varepsilon}$ is in $L^{1}\left(\Omega^{n}\right)$, and that $\left[\omega_{0}\right],[\Omega] \in N S_{\mathbb{R}}(X)$.

Let $\sigma_{1}, \ldots, \sigma_{p}$ (resp. $\tau_{1}, \ldots, \tau_{q}$ ) be holomorphic sections of some line bundle $L$ (resp $L^{\prime}$ ) on $X$. Fix $k \in \mathbb{R}_{\geq 0}, l \in \mathbb{R}_{\geq 0}$ and $F \in \mathcal{C}^{\infty}(X, \mathbb{R})$. Assume that

$$
\int_{X} \frac{1}{\left|\tau_{1}\right|^{2 l}+\ldots+\left|\tau_{q}\right|^{2 l}} \Omega^{n}<\infty \text { and } \int_{X} \frac{\left|\sigma_{1}\right|^{2 k}+\ldots+\left|\sigma_{p}\right|^{2 k}}{\left|\tau_{1}\right|^{2 l}+\ldots+\left|\tau_{q}\right|^{2 l}} e^{F} \Omega^{n}=\int_{X} \Omega^{n} .
$$

For each $t>0$, the unique function $\varphi \in P S H\left(X, \omega_{0}\right) \cap L^{\infty}(X)$ such that

$$
\left(\omega_{0}+d d^{c} \varphi\right)^{n}=\frac{\left|\sigma_{1}\right|^{2 k}+\ldots+\left|\sigma_{p}\right|^{2 k}}{\left|\tau_{1}\right|^{2 l}+\ldots+\left|\tau_{q}\right|^{2 l}} e^{F+t \varphi} \Omega^{n}
$$

is smooth outside $B=S \cup \bigcap_{i}\left\{\sigma_{i}=0\right\} \cup \bigcap_{i}\left\{\tau_{i}=0\right\}$.

Proof. The proof of Theorem 3.5 applies here almost verbatim.

Remark 4.6. We will apply Theorem 4.1 in Section 6 to construct singular KählerEinstein metrics on manifolds of general type. This will follow from the resolution of $\left(\omega+d d^{c} \varphi\right)^{n}=e^{t \varphi} \mu$ for large enough values of $t>0$. The Monge-Ampère equations

$$
\left(\omega+d d^{c} \varphi\right)^{n}=e^{-t \varphi} \mu, \quad t>0,
$$

can also be solved with a similar method, but only for small values of $t<t_{X}$. The critical exponent $t_{X}$ depends on the manifold $X$ and may be too small to produce Kähler-Einstein metrics when $c_{1}(X)>0$ : even smooth manifolds of positive scalar curvature do not necessarily admit Kähler-Einstein metrics (see [T]). Since technical details are much more involved in this case, we postpone this study to a forthcoming article.

\section{Singularities in Mori theory}

The singular locus of the normal complex space of pure dimension $n$ is a codimension $\geq 2$ analytic subvariety denoted by $V^{\text {sing }}$. Let $V^{r e g}=V-V^{\text {sing }}$ and let $j: V^{r e g} \rightarrow V$ be the natural open immersion. 
5.1. Log terminal singularities. Since this material may not be familiar to complex analysts or differential geometers, we briefly recall some basic facts on some of the singularities encountered in the Minimal Model Program (MMP for short). See [KM] for a detailled account in the algebraic case, the analytic theory being also surveyed there in less detail.

The sheaf of holomorphic functions $\mathcal{O}_{V}$ is the subsheaf of the sheaf of continuous functions on $V$ consisting of the functions whose restriction to $V^{r e g}$ is holomorphic. Actually, by Hartogs' theorem, any holomorphic function on $V^{\text {reg }}$ extends to $V$, which means that $j_{*} \mathcal{O}_{V^{\text {reg }}}=\mathcal{O}_{V}$.

Every meromorphic $n$-form $\alpha$ on $V^{r e g}$ extends to $V$; i.e. let $\pi: X \rightarrow V$ be a resolution of singularities of $V$, then the meromorphic $n$-form $\pi^{*} \alpha$ defined on $\pi^{-1} V^{r e g}$ extends to a meromorphic $n$-form on $X$. Let $\omega_{V^{r e g}}$ be the canonical sheaf of the smooth variety $V^{r e g}$. The sheaf $\omega_{V}=j_{*} \omega_{V^{r e g}}$ is a coherent analytic sheaf on $V$.

More generally every meromorphic pluricanonical form on $V^{\text {reg }}$ extends to $V$ and $\omega_{V}^{[q]}=j_{*} \omega_{V^{r e g}}^{q}, q>0$, is a coherent analytic sheaf on $V$.

Definition 5.1. Say $V$ is 1-Gorenstein iff one of the following equivalent conditions holds:

(1) Every $x \in V$ has an open neighborhood $U$ such that $U^{r e g}$ carries a holomorphic $n$-form with an empty zero divisor.

(2) $\omega_{V}$ is a rank one locally free sheaf.

(3) Every $x \in V$ has an open neighborhood $U$ such that $\omega_{U^{r e g}}$ is isomorphic to $\left.\mathcal{O}_{V^{r e g}}\right|_{U}$

A local section of $\omega_{V}$ defining a holomorphic $n$-form without zeroes on $V^{r e g}$ will be called a local generator of $\omega_{V}$. If furthermore $V$ is Cohen-Macaulay, $V$ is said to be Gorenstein.

Say $V$ is $\mathbb{Q}$-Gorenstein iff one of the following equivalent conditions is satisfied:

(1) Every $x \in V$ has an open neighborhood $U$ such that $U^{r e g}$ carries a holomorphic pluricanonical form with an empty zero divisor.

(2) For every $x \in V$, there exists $N_{x} \in \mathbb{N}$ and an open neighborhood $U$ of $x$ such that $\omega_{U}^{\left[N_{x}\right]}$ is a rank one locally free sheaf.

(3) For every $x \in V$ there is $N_{x} \in \mathbb{N}$ and an open neighborhood $U$ of $x$ such that $\omega_{U^{r e g}}^{N_{x}}$ is isomorphic to $\left.\mathcal{O}_{V^{r e g}}\right|_{U}$.

A local section of $\omega_{V}^{[N]}$ defining a holomorphic pluricanonical form without zeroes on $V^{\text {reg }}$ will be called a local generator of $\omega_{V}^{[N]}$.

For every $x \in V$, the smallest $N_{x}$ fulfilling condition (3) near $x$ is called the local index of $V$ at $x$. The l.c.m. of all local indices, if finite, is called the index of $V$.

Definition 5.2. Say $V$ has only canonical singularities iff $V$ is $\mathbb{Q}$-Gorenstein, of finite index $N$ and one of the following equivalent conditions is fulfilled:

(1) Let $\pi: X \rightarrow V$ be a resolution. Let $\alpha$ be a local generator of $\omega_{V}^{[N]}$. The meromorphic pluricanonical form $\pi^{*} \alpha$ is holomorphic.

(2) Let $\pi: X \rightarrow V$ be a resolution. For every $m \in \mathbb{N}, \pi_{*} \omega_{X}^{[N m]}=\omega_{V}^{[N m]}$.

(3) (Assuming $V$ is an algebraic variety) Let $\pi: X \rightarrow V$ be a resolution. Then $K_{X} \cong \pi^{*} K_{V}+\sum a_{E} E$ with $a_{E} \geq 0$ where $\cong$ means numerical equivalence of $\mathbb{Q}$-Cartier divisors and the sum runs over the exceptional divisors of $\pi$. 
Observe that it is enough to check the first two conditions for some resolution. In the third condition $N a_{E}$ is the order of vanishing of $\pi^{*} \alpha$ along the divisor $E$.

Definition 5.3. Say $V$ has only log-terminal singularities iff $V$ is $\mathbb{Q}$-Gorenstein, of finite index $N$ and the following holds. Let $\pi: X \rightarrow V$ be a log-resolution and let $\alpha$ be a local generator of $\omega_{V}^{[N]}$; then the pole along any component $E$ of $\operatorname{exc}(\pi)$ of the meromorphic $N$-canonical form $\pi^{*} \alpha$ on $X$ is of order $\leq N-1$.

When $V$ is algebraic, an equivalent formulation is: let $\pi: X \rightarrow V$ be a logresolution. Then $K_{X} \cong \pi^{*} K_{V}+\sum_{E} a_{E} E$ with $a_{E}>-1$.

The importance of the class of canonical singularities 12 comes from a theorem due to M. Reid [R1] (see also Deb], p. 174):

Theorem 5.4. Let $X$ be a projective algebraic manifold of general type whose canonical ring $R=\bigoplus_{n \in \mathbb{N}} H^{0}\left(X, \omega_{X}^{n}\right)$ is of finite type. Then the canonical model of $X, X_{\text {can }}:=\operatorname{Proj}(R)$, has only canonical singularities. If $N=\operatorname{Index}\left(X_{\text {can }}\right)$, then $\omega_{X_{\text {can }}}^{[N]}$ is ample.

The finiteness of the canonical ring for varieties of general type is known in dimension $3[\mathrm{Ka}$. In higher dimension, Y. Kawamata has proved that it is a consequence of the existence of minimal models. $X_{c a n}$ is a uniquely defined singular birational model of $X$. The minimal models of $X$ in the sense of the MMP are crepant terminalizations of $X_{\text {can }}$ and do not enjoy the above unicity since they may be related by non-trivial flops.

Examples 5.5. Let $S$ be a normal algebraic surface. The following are equivalent:

(1) $S$ has only canonical singularities.

(2) $S$ is locally analytically isomorphic to $X=\mathbb{C}^{2} / G, G \subset S L_{2}(\mathbb{C})$ a finite subgroup.

(3) The exceptional divisors of the minimal resolution $\pi_{\min }$ of $S$ have simple normal crossings, their components are $(-2)$ smooth rational curves, their incidence graphs are of type $A-D-E$ (Du Val singularities).

The log-terminal surface singularities are precisely the singularities of the form $X=\mathbb{C}^{2} / G, G \subset G L_{2}(\mathbb{C})$ a finite subgroup.

Examples 5.6. In higher dimension, quotient singularities are still log-terminal. Fix $n>0$ and let $H \subset \mathbb{C P}^{n+1}$ be a smooth degree $d$ hypersurface. The affine cone over $H$ has only canonical singularities iff $d \leq n+1$.

In particular, the ordinary double point $x^{2}+y^{2}+z^{2}+t^{2}=0$ has only canonical singularities but it is not a quotient singularity.

The hypersurface singularities of type $A-D-E$ are canonical.

\footnotetext{
${ }^{12} \mathrm{On}$ the other hand, the class of log-terminal singlarities of varieties is less important. Indeed let $X$ be a complex projective normal variety with log-terminal singularities. Then there is a Deligne Mumford stack $\mathcal{X} \rightarrow X$ which is etale in codimension one and has only Gorenstein canonical singularities. At the expense of working with this canonical cover, one could avoid the consideration of log-terminal singularities for varieties.
} 


\subsection{Normal Kähler spaces.}

Plurisubharmonic functions. Let $V$ be a normal analytic space of pure dimension $n$. A plurisubharmonic (psh) function $\varphi$ on $V$ is an upper-semi-continuous function on $V$ with values in $\mathbb{R} \cup\{-\infty\}$, which is not locally $-\infty$, and extends to a psh function in some local embedding $V \rightarrow \mathbb{C}^{N}$. The function $\varphi$ is strongly psh (resp. $\mathcal{C}^{0}$, resp. $\mathcal{C}^{\infty}$ ) iff it extends to a strongly psh function (resp. $\mathcal{C}^{0}$, resp. $\mathcal{C}^{\infty}$ ) in some local embedding. A continuous function is psh iff its restriction to $V^{\text {reg }}$ is so [FN]. A bounded psh function on $V^{\text {reg }}$ extends to $V$.

A pluriharmonic function $f$ on $V$ is a real-valued continuous function such that one of the following equivalent conditions holds:

- $f$ is locally the real part of a holomorphic function.

- Given a local embedding $V \rightarrow \mathbb{C}^{N}, f$ extends locally to a pluriharmonic function on $\mathbb{C}^{N}$.

- $\left.f\right|_{V^{r e g}}$ is pluriharmonic.

Semi-Kähler currents.

Definition 5.7. A semi-Kähler, resp. Kähler, resp. smooth Kähler, potential on $V$ is a family $\left(U_{i}, \varphi_{i}\right)_{i \in I}$ where $\left(U_{i}\right)$ is an open covering of $V$ and $\varphi_{i}$ is a psh function, resp. a strongly psh function, resp. a $\mathcal{C}^{\infty}$-smooth strongly psh function, on $U_{i}$ such that $\varphi_{i}-\varphi_{j}$ is pluriharmonic on $U_{i} \cap U_{j}$.

Define an equivalence relation on semi-Kähler potentials requiring that $\left(U_{i}, \varphi_{i}\right) \sim$ $\left(V_{j}, \psi_{j}\right)$ iff $\varphi_{i}-\psi_{j}$ is pluriharmonic on $U_{i} \cap V_{j}$.

Definition 5.8. A smooth Kähler metric $\Omega$ on $V$ is a $\sim$-equivalence class of smooth Kähler potentials. A semi-Kähler (resp. Kähler) current on $V$ is a -equivalence class of semi-Kähler (resp. Kähler) potentials.

A semi-Kähler current $\Omega$ is said to have $L_{\text {loc }}^{\infty}$ (resp. $\mathcal{C}^{0}$, resp. Hölder continuous) potentials iff, given a potential $\left(U_{i}, \varphi_{i}\right)_{i \in I}$ for $\Omega$, each $\varphi_{i}$ is $L_{l o c}^{\infty}$ (resp. $\mathcal{C}^{0}$, resp. Hölder continuous).

We will on occasion drop the requirement that the local potentials of $\Omega$ are psh, replacing it by the requirement that they are locally the sum of a smooth and a psh function. The current $\Omega$ will then be called a quasi-positive closed current on $V$.

If it has locally bounded potentials, $\Omega$ is fully determined by the closed $(1,1)$ form $\Omega_{\text {reg }}$ on $V_{\text {reg }}$ defined on $U_{i}$ by $\Omega_{r e g}=d d^{c} \varphi_{i}$.

Let $\Omega$ be a smooth Kähler metric on $V$ with Kähler potential $\left(U_{i}, \varphi_{i}\right)$. An uppersemi-continuous function $\varphi: X \rightarrow \mathbb{R} \cup-\infty$ is said to be $\Omega$-psh iff $\forall i \varphi_{i}+\varphi$ is psh on $U_{i}$. The semi-Kähler current whose potential is $\left(U_{i}, \varphi+\varphi_{i}\right)$ is denoted by $\Omega+d d^{c} \varphi$.

Example 5.9. Let $V=\mathbb{C}^{2} / \pm 1$. Let $(x, y)$ be the usual affine coordinates on $\mathbb{C}^{2}$, $(u, v, w)$ those on $\mathbb{C}^{3}$. The formulas $u=x^{2}, v=y^{2}, w=x y$ realize $V$ as the closed subscheme of $\mathbb{C}^{3}$ whose equation is $u v-w^{2}=0$. We have two 'natural' Kähler metrics on $V$. The first one is smooth with potential $\varphi^{1}=|u|^{2}+|v|^{2}+|w|^{2}$, induced by the euclidean Kähler metric of $\mathbb{C}^{3}$; the second one is the Kähler current whose potential is $\varphi^{2}=|u|+|v|$. On $V^{\text {reg }}$ it is the quotient of the euclidean metric restricted to $\mathbb{C}^{2}-\{0\}$. Near $0, d d^{c} \varphi^{2} \gg d d^{c} \varphi^{1}$. 
The metric $d d^{c} \varphi^{2}$ is an example of an orbifold Kähler metric on $V$. The results of [Y] extend without major modifications to Kähler orbifolds. For instance, in each Kähler class of a nodal K3 surface there is a unique Ricci flat orbifold metric.

Chern-Weil forms and hermitian metrics. Let $\mathcal{P} \mathcal{H}_{V}$ be the sheaf of real-valued pluriharmonic functions on $V$. By definition, a closed (1,1)-form on $V$ is a section of the sheaf $\mathcal{C}_{V}^{\infty} / \mathcal{P} \mathcal{H}_{V}$. We have the exact sequence:

$$
\mathcal{C}^{\infty}(V) \rightarrow \Gamma\left(V, \mathcal{C}_{V}^{\infty} / \mathcal{P} \mathcal{H}_{V}\right) \stackrel{[\cdot]}{\longrightarrow} H^{1}\left(V, \mathcal{P} \mathcal{H}_{V}\right) \rightarrow 0 .
$$

A class in $H^{1}\left(X, \mathcal{P} \mathcal{H}_{X}\right)$ will be called Kähler if it is in the [ . ] image of a smooth Kähler metric.

Remark 5.10. Assume $X$ is smooth. A class $[\omega]$ in $H^{1}\left(X, \mathcal{P} \mathcal{H}_{X}\right)$ will be called numerically base point free iff there exists a proper surjective holomorphic mapping $X \rightarrow Y, Y$ normal, such that $[\omega]$ is the pull back of a Kähler class on $Y$. This is in principle a stronger condition than being cohomologous to a smooth semi-positive closed $(1,1)$-form, although no counterexample seems to be known.

If $X$ is projective and $[\omega] \in N S_{\mathbb{R}}(X),[\omega]$ is numerically a base point free class iff it is semi-ample.

In the non-big case (i.e. $\int_{X} \omega^{n}=0$ ), it is straightforward to construct semiKähler classes that are not numerically base point free (e.g. on complex tori).

Let $L$ be a holomorphic line bundle on $V$. The notion of smooth hermitian metric on $(V, L)$ is defined as in the smooth case. Let $h$ be such a metric on $(V, L)$.

Let $s \in H^{0}(U, L)$ be a nowhere zero local holomorphic section of $L$ (a local generator of $L$ ) defined over the open subset $U \subset V$. Set $e^{-\varphi_{s}}:=\|s\|_{h}^{2}$, where $\varphi_{s}$ is a $\mathcal{C}^{\infty}$-smooth function on $U$. The current $d d^{c} \varphi_{s}$ is a smooth closed $(1,1)$-form on $V$ which does not depend on $s$; it is a semi-Kähler current if $\varphi_{s}$ is psh.

More generally, let $\left(U_{i}\right)_{i}$ be an open covering of $V$ and $\sigma_{i} \in H^{0}\left(U_{i}, \mathcal{O}_{V}(L)\right)$ a local generator of $L$. Let $\varphi_{i}=\varphi_{\sigma_{i}}$. The datum $\left(U_{i}, \varphi_{i}\right)$ defines a smooth closed (1,1)-form on $V$.

Definition 5.11. The Chern-Weil form of $(V, L, h)$ (or of $h$ ) is the $\sim$-equivalence class of the data $\left(U_{i}, \varphi_{i}\right)$ constructed above. We will denote it by $c_{1}(L, h)$.

It is immediate that $\left[c_{1}(L, h)\right]$ is independent of $h$. Hence there is a linear map $c_{1}: \operatorname{Pic}(V) \rightarrow H^{1}\left(V, \mathcal{P} \mathcal{H}_{V}\right)$. The connection with the more widely known smooth case is made by the observation that, if $X$ is a compact Kähler manifold, $H^{1,1}(X, \mathbb{R})=H^{1}\left(X, \mathcal{P} \mathcal{H}_{X}\right)$.

Proposition 5.12. Let $V$ a compact normal complex analytic variety.

The space $H^{1}\left(V, \mathcal{P} \mathcal{H}_{V}\right)$ is finite dimensional.

Let $L$ be a holomorphic line bundle on $V$. Every representative of $c_{1}(L)$ in $H^{1}\left(V, \mathcal{P} \mathcal{H}_{V}\right)$ is the Chern-Weil form of a smooth hermitian on $L$.

If there exists a smooth hermitian metric $h$ such that $c_{1}(L, h)$ is Kähler, then $V$ is projective-algebraic and $L$ is ample.

Proof. The most difficult task is to show that, in the last assertion, $V$ is Moishezon. This follows from Siu's solution of the Grauert-Riemenschneider conjecture [Siu]. 
A singular metric on $L$ is an expression $h=e^{-\varphi} h_{s m}, \varphi$ being a locally smooth + psh function and $h_{s m}$ a smooth hermitian metric. Its Chern-Weil form is the quasi-positive current $c_{1}\left(L, h_{s m}\right)+d d^{c} \varphi$.

\section{Adapted volume forms}

6.1. Monge-Ampère equations on normal Kähler spaces. Let $\Omega$ be a smooth Kähler metric on $V$. A classical result of $\mathrm{P}$. Lelong states that if $U$ is relatively compact in $V$, then $U^{r e g}$ is of finite volume with respect to the smooth volume form $\Omega_{r e g}^{n}$.

This has been generalized by E. Bedford and B. A. Taylor in [BT], where the authors study Monge-Ampère measures for locally bounded psh functions. Since these measures do not charge proper analytic subsets, we obtain

Proposition 6.1. Let $\Omega$ be a semi-Kähler current with $L_{\text {loc }}^{\infty}$ potentials on $V$. The Monge-Ampère measure $\Omega_{r e g}^{n}$ is well defined on $V_{\text {reg }}$ and satisfies $\int_{U^{r e g}} \Omega_{r e g}^{n}<$ $\infty$, for all relatively compact subsets $U \subset V$.

For any resolution $\pi: X \rightarrow V$, the Monge-Ampère measure $\left(\pi^{*} \Omega\right)^{n}$ is well defined on $X$ and satisfies $\pi_{*}\left(\pi^{*} \Omega\right)^{n}=j_{*} \Omega_{r e g}^{n}$. Moreover if $\bar{\pi}: \bar{X} \rightarrow V$ is a resolution dominating $\pi$ (i.e. $\bar{\pi}=\pi \circ \psi$ for some bimeromorphic proper holomorphic map $\left.\psi: \bar{X}^{\prime} \rightarrow X^{\prime}\right)$, then $\psi_{*}\left(\bar{\pi}^{*} \Omega\right)^{n}=\left(\pi^{*} \Omega\right)^{n}$.

The measure $\pi_{*}\left(\pi^{*} \Omega\right)^{n}$ is thus well defined on $V$ and independent of the choice of resolution. We will call it the Monge-Ampère measure of $\Omega$ and denote it by $\Omega^{n}$. The mass of this measure only depends on the cohomology class of $\Omega$, as follows again from $\mathrm{BT}$ :

Lemma 6.2. Assume $V$ is compact. Let $\Omega_{1}, \Omega_{2}$ be two semi-Kähler currents with $L_{\text {loc }}^{\infty}$ potentiel on $V$. If they are cohomologous, i.e. $\Omega_{1}=\Omega_{2}+d d^{c} \varphi$ for some $\varphi \in L^{\infty}(X)$, then $\int_{V} \Omega_{1}^{n}=\int_{V} \Omega_{2}^{n}$.

We can now reformulate some of our previous results.

Theorem 6.3. Let $V$ be an $n$-dimensional compact normal Kähler space and let $\Omega$ be a smooth Kähler form on $V$. Then for every $f \in L^{p}\left(V, \Omega^{n}\right), p>1$, such that $\int_{V} f \Omega^{n}=\int_{X} \Omega^{n}$, there is a unique $\varphi \in L^{\infty}(V)$ such that

$$
\left(\Omega+d d^{c} \varphi\right)^{n}=f \Omega^{n} \text { and } \sup _{V} \varphi=-1 .
$$

Proof. Let $\pi: X \rightarrow V$ be a resolution of $V$. We may define a semi-positive big smooth form on $X$ by $\omega=\pi^{*} \Omega$. By Theorem 2.1 and Proposition 3.1 we can solve uniquely $\left(\omega+d d^{c} \bar{\varphi}\right)^{n}=f \circ \pi \omega^{n}$ where $\bar{\varphi}$ is a continuous function on $X$ such that $\omega+d d^{c} \bar{\varphi}$ is semi-positive. Let $F$ be a fiber of $\pi$ and $i: F \rightarrow X$ the inclusion map. The fiber $F$ is connected by Zariski's main theorem. Furthermore $i^{*} \omega+d d^{c} i^{*} \bar{\varphi}$ is semi-positive on $F$. Since $i^{*} \omega=0$, it follows that $i^{*} \varphi$ is a psh function on $F$. Hence $i^{*} \bar{\varphi}$ is constant. This implies that $\bar{\varphi}=\varphi \circ \pi$ where $\varphi$ is a bounded u.s.c. function on $V$. We do have $\left(\Omega+d d^{c} \varphi\right)^{n}=f \Omega^{n}$.

6.2. Adapted measures on log-terminal Kähler spaces. Let $V$ be an $n$ dimensional Gorenstein Kähler space and let $\Omega$ be a smooth Kähler form on $V$. Fix $x \in V$ and let $\alpha$ be a local generator of $\omega_{V}$ defined over an open subset $x \in U$; then $v=c_{n} \alpha \wedge \bar{\alpha}$ is a positive definite volume form on $U^{r e g}$, for an appropriate choice of the constant $c_{n}=\sqrt{-1}^{n}(-1)^{\frac{n(n+1)}{2}}$. 
When $V$ is merely $\mathbb{Q}$-Gorenstein of finite index $N$, we choose $\beta$ a local generator of $\omega_{V}^{[N]}$ defined over an open subset $x \in U$ and we set

$$
v=v_{\beta}=\left(\sqrt{-1}^{N n}(-1)^{N \frac{n(n+1)}{2}} \beta \wedge \bar{\beta}\right)^{\frac{1}{N}} .
$$

This is a positive definite volume form on $U^{r e g}$.

Our next observation is that log-terminal singularities are the worst singularities we can allow in order to globally solve Monge-Ampère equations associated to volume forms on $V$.

Lemma 6.4. For every $U_{1} \subset \subset U, \int_{U_{1}^{r e g}} v<\infty$ iff $X$ is log-terminal.

If $V$ is log-terminal, then the Radon measure $\mu=j_{*} v$ satisfies $\mu=f \Omega^{n}$ with $f \in L^{1+\varepsilon}\left(U_{1}, \Omega^{n}\right)$ for some $\varepsilon>0$.

Proof. Let $\pi: X \rightarrow V$ be a log-resolution. Write $K_{X} \cong \pi^{*} K_{V}+\sum a_{E} E$. Since $\operatorname{exc}(\pi)$ has simple normal crossings, at every $P \in E=\operatorname{exc}(\pi)$ there are local coordinates $\left(z^{i}\right)_{i=1, \ldots, n}$ such that $E$ is described by the equation $z^{1} \ldots z^{q}=0$. Let $E_{j}$ be the divisor $z_{j}=0$. We have $\pi^{*} v=\prod_{j=1}^{q}\left|z^{j}\right|^{2 a_{E_{j}}} d \lambda$ where $d \lambda$ is a Lebesgue measure on $X$; hence the measure $\pi^{*} v$ has finite mass near $P$ iff $\forall j, a_{E_{j}}>-1$. Thus $\int_{U_{1}^{r e g}} v<\infty$ iff $\forall E, a_{E}>-1$.

Let $f_{1}$ be the density of $\pi^{*} v$ with respect to $d \lambda$. Since $f_{1}$ is comparable to $\prod_{j=1}^{q}\left|z^{j}\right|^{2 a_{E_{j}}}$ near $P$, it follows that $f_{1}$ belongs actually to $L^{p}(X, d \lambda)$ for some $p>1$ when $X$ is log-terminal.

Let $D=1 / f$ be the density of $\Omega^{n}$ with respect to $v$. We will see here below that $D$ is bounded but it might have zeroes on $E$; hence $f$ is unbounded in general. However we will show that $f \circ \pi \in L^{\alpha}(X, d \lambda)$ for $\alpha>0$ small enough; hence it follows from Hölder's inequality (as in the proof of Lemma 3.2) that

$$
\int_{U_{1}^{r e g}} f^{1+\varepsilon} \Omega^{n}=\int_{\pi^{-1} U_{1}^{r e g}} f^{\varepsilon} f_{1} d \lambda<+\infty
$$

if $\varepsilon>0$ is small enough.

Fix $x \in V$ and let $i: U_{x} \rightarrow \mathbb{C}^{m}$ be a local embedding of a neighborhood $U_{x}$ of $x$. We consider the $\left(\begin{array}{c}m \\ n\end{array}\right) n$-forms on $U_{x}^{r e g} d u^{I}=d u^{i_{1}} \wedge \ldots d u^{i_{n}}$, where $\left(u^{i}\right)$ is a set of affine coordinates on $\mathbb{C}^{m}$. Observe that $\Omega^{n}$ is comparable to $\sum_{I} v_{d u^{I}} 13$ Since $\beta$ is a local generator at $x$ of $\omega_{V}^{[N]}$, we have $\left(d u^{I}\right)^{N}=f_{I} \beta$ where $f_{I} \in \mathcal{O}_{V, x}$ is the germ of a holomorphic function at $x$. Therefore $\Omega^{n}$ is comparable to $\sum_{I}\left|f_{I}\right|^{\frac{2}{N}} v$; hence $D$ is comparable to $\left[\sum_{I}\left|f_{I}\right|^{\frac{2}{N}}\right]^{-1}$ near $x$.

The functions $\left(f_{I}\right)$ generate an ideal $\mathcal{I}_{x} \subset \mathcal{O}_{V, x}$. Actually, the construction can be globalized to provide a coherent ideal sheaf $\mathcal{I} \subset \mathcal{O}_{V}$ cosupported on $V^{\text {sing }}$.

We may assume [Hi], $\mathrm{BM}$ that $\pi: X \rightarrow V$ is a log-resolution of $(V, \mathcal{I})$, namely a $\log$-resolution of $V$ with the additional property that the ideal sheaf $\pi^{-1} \mathcal{I} \cdot \mathcal{O}_{X}$, which is the ideal sheaf of $\mathcal{O}_{X}$ generated by the family of holomorphic functions $\left(\pi^{*} f_{I}\right)_{I}$, satisfies $\pi^{-1} \mathcal{I} \cdot \mathcal{O}_{X}=\mathcal{O}_{X}\left(-\sum N b_{E} E\right) \subset \mathcal{O}_{X}$ where $N . b_{E} \in \mathbb{N}$ is a positive multiplicity attached to any exceptional divisor of $\pi$.

In local coordinates near $P \in X, \pi^{*} D$ is comparable to $\prod_{j}\left|z_{j}\right|^{2 b_{E_{j}}}$; hence $\pi^{*}\left(f_{1} D^{-\varepsilon}\right)$ is comparable to $\prod_{j}\left|z_{j}\right|^{2\left(a_{E_{j}}-\varepsilon b_{E_{j}}\right)}$. It follows that for every relatively

\footnotetext{
${ }^{13}$ Note that the formula for $v_{\beta}$ makes sense even if $\beta$ is not a local generator.
} 
compact subset $U_{1} \subset \subset U, \quad f \in L^{1+\varepsilon}\left(U_{1}, \Omega^{n}\right)$ iff $\forall E, \pi(E) \cap U \neq \emptyset \Rightarrow a_{E}-\varepsilon b_{E}>$ -1 .

Definition 6.5. Assume $V$ has only log-terminal singularities. A positive definite adapted measure on $V$ is a positive Radon measure locally of the form $e^{f} . v$ where $f$ is a bounded measurable function. A positive definite adapted measure has $\mathcal{C}^{0}$, $\mathcal{C}^{\alpha}, \mathcal{C}^{\infty}$ density if $f$ is such.

Remark 6.6. It follows from Lemma 6.4 that if $V$ is $\mathbb{Q}$-Gorenstein but has non-logterminal singularities, $v$ is a volume form on $V^{r e g}$ but does not extend to a measure on $V$.

6.3. Adapted volume forms for klt Kähler pairs. We will be briefer since pairs are mainly of interest to MMP practitioners. The key definition for us will be

Definition 6.7. A pair $(V, \Delta)$ is klt iff $K_{V}+\Delta$ is $\mathbb{Q}$-Cartier and if for any logresolution $\pi: X \rightarrow V$ of $(V, \Delta)$, we have the numerical equivalence of Cartier divisors:

$$
N\left(K_{X}+\Delta^{\prime}\right) \cong \pi^{*} N\left(K_{V}+\Delta\right)+\sum_{E \text { exc. }} N a_{E} E
$$

with $a_{E}>-1, \Delta^{\prime}$ the proper transform of $\Delta$ in $X$ (same multiplicities) and $N$ is an integer such that $N\left(K_{V}+\Delta\right)$ is Cartier.

Thus a variety $V$ has only klt singularities iff $(X, \emptyset)$ is klt.

Let $\beta$ be a local generator of $\mathcal{O}_{V}\left(N\left(K_{V}+\Delta\right)\right)$. Then $\beta_{V}$ reg can be viewed as a meromorphic $N$-canonical form with a pole of order $N d_{i}$ on $E_{i}$ where $\Delta=\sum_{i} d_{i} E_{i}$ is the decomposition of $\Delta$ into prime divisors. Thus $v_{\beta_{V} \text { reg }}$ defines a volume form with poles on $V^{r e g}$; namely $v_{\beta_{V^{r e g}}}$ is comparable to $\prod_{i}\left|\sigma_{i}\right|^{-2 d_{i}} d \lambda$, where $\sigma_{i}$ denotes the canonical section of $\mathcal{O}\left(E_{i}\right)$. If $v_{\beta_{V} \text { reg }}$ is a finite measure, then $d_{i}<1$, but the converse is not true. We have the following staightforward extension of Lemma 6.4

Lemma 6.8. Let $j^{\prime}: V-\bigcup_{i} E_{i} \rightarrow V$ be the canonical inclusion. Then $j_{*}^{\prime} v_{\beta}$ is a well defined Radon measure on $V$ iff $(X, \Delta)$ is klt.

The definition of an adapted measure for a klt pair is left to the reader.

\section{Singular KähleR-EINSTEIN METRICS}

\subsection{Singular Ricci curvature.}

The smooth case. The link between Monge-Ampère equations and Kähler-Einstein metrics is provided by the following classical

Lemma 7.1. Let $X$ be a complex manifold, let $h$ be a smooth hermitian metric on $\omega_{X}$ and let $\Omega$ be a Kähler form such that $\Omega^{n}=v(h)$. The Ricci curvature divided by $2 \pi$ of $\Omega$ is the Chern-Weil form $-c_{1}\left(K_{X}, h\right)$.

Adapted measures and hermitian metrics on the canonical sheaf. Assume $V$ is compact with only canonical singularities and that it has index $N$ and let $h^{N}$ be a smooth hermitian metric on $\omega_{V}^{[N]}$. Let $\beta$ be a local generator of $\omega_{V}^{[N]}$. Define $v_{\beta}(h)$ to be the volume form on $V^{r e g}$ :

$$
v_{\beta}(h)=\left(\sqrt{-1}^{N n}(-1)^{N \frac{n(n+1)}{2}} \frac{\beta \wedge \bar{\beta}}{\|\beta\|_{h^{N}}^{2}}\right)^{\frac{1}{N}} .
$$


Since $v_{\beta}(h)$ is independent of $\beta$, this expression defines an adapted measure $v(h)$ with $\mathcal{C}^{\infty}$ density on $V$.

Now, let $h_{\text {sing }}^{N}=e^{-N \chi} h^{N}$ be a singular metric on $\omega_{V}^{[N]}$. The Chern-Weil form $c_{1}\left(\omega_{X}^{N}, h_{\text {sing }}^{N}\right)$ is then well defined as a quasi-positive current. Since $h_{\text {sing }}^{N}$ has locally $\mathcal{C}^{\infty}+$ psh potentials, $\chi$ is locally bounded above and the above formula defines a measure $v\left(h_{\text {sing }}\right)=e^{\chi} v(h)$ on $V$ such that $\frac{v\left(h_{\text {sing }}\right)}{v(h)} \in L_{l o c}^{\infty}$. In particular

$$
\frac{v\left(h_{\text {sing }}\right)}{\Omega^{n}} \in L^{1+\varepsilon}\left(V, \Omega^{n}\right) \text { for } \varepsilon>0 \text { small enough. }
$$

We have $c_{1}\left(K_{X}, h_{\text {sing }}\right)=c_{1}\left(K_{X}, h\right)+d d^{c} \chi$, where $c_{1}\left(K_{X}, h\right):=\frac{1}{N} c_{1}\left(\omega_{X}^{N}, h^{N}\right)$.

Definition 7.2. Assume $V$ has only canonical singularities. An adapted measure on $V$ is a positive Radon measure locally of the form $e^{f} \cdot v$ where $f$ is locally given as the sum of a psh and a smooth function on $V$. An adapted measure has $\mathcal{C}^{0}$, resp. $\mathcal{C}^{\alpha}$, resp. $\mathcal{C}^{\infty}$, density if $e^{f}$ is $\mathcal{C}^{0}$, resp. $\mathcal{C}^{\alpha}$, resp. $\mathcal{C}^{\infty}$.

The definition has the virtue of generalizing the usual equivalence between smooth metrics on the canonical sheaf of a manifold and positive definite volume forms to singular metrics and log-terminal spaces. This suggests the following:

Definition 7.3. Let $V$ be a $\mathbb{Q}$-Gorenstein Kähler normal $n$-dimensional complex space with only log terminal singularities. Let $\Omega$ be a semi-Kähler current with $L_{l o c}^{\infty}$ potential and adapted Monge-Ampère measure. Let $h$ be the singular metric on the canonical sheaf such that $\Omega^{n}=v(h)$. We define

$$
\operatorname{Ric}(\Omega):=-c_{1}\left(K_{V}, h\right),
$$

where the equality is to be taken in the sense of currents.

Here $\Omega$ will be called a singular Kähler-Einstein metric if $\operatorname{Ric}(\Omega)=c \Omega$ for some $c \in \mathbb{R}$.

\subsection{Singular Ricci flat metrics.}

Definition 7.4. Let $V$ be a Kähler space with only canonical singularities. The space $V$ is said to be $\mathbb{Q}$-CY iff there is some multiple $N^{\prime}$ of index $(X)$ such that $H^{0}\left(V, \omega_{V}^{\left[N^{\prime}\right]}\right)=\mathbb{C} \alpha$, where $\alpha$ is a global generator of $\omega_{V}^{\left[N^{\prime}\right]}$.

Theorem 7.5. Assume $V$ is a compact $\mathbb{Q}$-CY Kähler space. Let $\Omega$ be a smooth Kähler metric on $V$. Then there is a unique semi-Kähler current with locally bounded potential and adapted Monge-Ampère measure $\Omega^{\prime}=\Omega+d d^{c} \varphi$, such that

$$
\left(\Omega+d d^{c} \varphi\right)^{n}=C v_{\alpha} \text { and } \sup _{V} \varphi=-1,
$$

where $\int_{V} \Omega^{n}=C \int_{V}(-1)^{n} v_{\alpha}$.

Furthermore, if $V$ is projective-algebraic and $[\Omega] \in N S_{\mathbb{R}}(V)$, then $\Omega+d d^{c} \varphi$ is smooth on $V^{\text {reg }}$ where it defines a bona fide Ricci flat metric.

Corollary 7.6. In each cohomology class of a smooth Kähler form, there is a unique singular Ricci flat metric.

Proof. This follows straighforwardly from Theorems 6.3, 3.5, Lemma 6.4 and Definition 7.3 .

Example 7.7. A nodal quintic threefold is $\mathbb{Q}$-CY and does not have quotient singularities, so the orbifold method of [Kö] does not work. 


\subsection{Singular Kähler-Einstein metrics of negative curvature.}

Theorem 7.8. Let $V$ be a general type projective algebraic variety with only canonical singularities such that $K_{V}$ is ample. Let $h^{N}$ be a smooth hermitian metric on $\omega_{V}^{N}$ such that $\Omega=c_{1}\left(K_{V}, h\right)$ is a smooth Kähler form on $V$.

There is a unique $\varphi \in L^{\infty}(V, \mathbb{R})$ such that

(1) $\varphi$ is $\Omega$-psh.

(2) $\Omega+d d^{c} \varphi$ is a semi-Kähler current with $L^{\infty}$ potentials.

(3) $\left(\Omega+d d^{c} \varphi\right)^{n}=e^{\varphi} v(h)$.

Consequently $\Omega+d d^{c} \varphi$ is the unique singular KE metric on $V$ of negative curvature in the canonical class of $V$. The current $\Omega+d d^{c} \varphi$ has locally bounded potentials and is smooth on $V^{\text {reg }}$ where it defines a bona fide KE metric.

Proof. This is a consequence of Theorem 4.1. Proposition 4.4, and Definition 7.3.

Remark 7.9. Thanks to Theorem [5.4, for $X$ a projective algebraic manifold of general type such that $R(X):=\bigoplus_{n \in \mathbb{N}} H^{0}\left(X, \mathcal{O}_{X}\left(n K_{X}\right)\right)$ is finitely generated, $X$ has a unique birational model $V$ such that the above hypotheses hold. Thus we have a birational map $\pi: X \rightarrow V$ which is well defined outside an indeterminacy locus $S$ of codimension $\leq 2$. In particular $\pi^{*}\left(\omega+d d^{c} \varphi\right)$ is a closed positive current on $X-S$ that extends to a closed positive current $T$ on $X$ itself. The current $T$ defines a KE metric on $X-S$. It does not need to be a singular KE metric on $X$ though, since its potentials may have logarithmic poles on $S$, in fact algebraic singularities of the form $\alpha \log \left(\sum\left|f_{i}\right|^{2}\right)+O(1), f_{i}$ holomorphic and $\alpha \in \mathbb{Q}_{>0}$. Moreover, $T$ lies in the canonical class of $X$ iff $X$ is a smooth minimal model as in Ts.

Connection with $\overline{\mathrm{Ts}}$. Let $X$ be a complex projective manifold such that $K_{X}$ is nef and big. Let $\Omega$ be a smooth Kähler metric on $X$ and consider the Kähler-Ricci flow

$$
\frac{\partial \Omega_{t}}{\partial t}=-\operatorname{Ric}\left(\Omega_{t}\right)-\Omega_{t}, \quad \Omega_{0}=\Omega
$$

In $[\mathrm{Ts}$, it was proved that this flow has a global solution for all time $t \in[0, \infty[$, and an argument was given, recently fully completed in [TZ, to the effect that $\Omega_{t}$ converges to a closed positive current $T_{K E}$, independent of $\Omega$, which defines a smooth Kähler-Einstein metric outside the exceptional divisor $E$ of the holomorphic bimeromorphic map $X \rightarrow X_{c a n}$. Its potential satisfies the Monge Ampère équation considered in Theorem $[7.8$ outside E. It follows from Proposition 4.4 that the current $T_{K E}$ coincides with the solution produced by Theorem 7.8.

The independant work $[\mathrm{TZ}$ gives a proof of the following properties, already conjectured by $\left[\mathrm{TS}\right.$, that $T_{K E}$ has locally bounded potential and satisfies the degenerate Monge-Ampère equation considered in Theorem 7.8 .

Example 7.10. A nodal sextic threefold is of general type, Gorenstein, terminal, is its own canonical model, has no smooth minimal model and does not have quotient singularities. Therefore the orbifold method of [Kö] does not work and [Ts] does not apply.

7.4. Singular KE metrics on klt pairs. Let us now state the immediate generalization to klt pairs.

Definition 7.11. Let $(V, \Delta)$ be a klt compact Kähler pair. 
The pair $(V, \Delta)$ is said to be $\mathbb{Q}$-CY iff there is some multiple $N^{\prime}$ of $\operatorname{index}(X, \Delta)$ such that

$$
H^{0}\left(V, \mathcal{O}_{V}\left(N^{\prime}\left(K_{V}+\Delta\right)\right)\right)=\mathbb{C} \alpha
$$

where $\alpha$ is a global generator of $\mathcal{O}_{V}\left(N^{\prime}\left(K_{V}+\Delta\right)\right)$.

The pair $(V, \Delta)$ is canonically polarized iff $K_{V}+\Delta$ is ample.

Theorem 7.12. Let $(V, \Delta)$ be a klt compact Kähler pair.

If $(V, \Delta)$ is $\mathbb{Q}-C Y$, it carries a singular Ricci flat metric with adapted volume form in any Kähler class of $V$, this current being smooth outside $\Delta \cup V^{\text {sing }}$ if $V$ is projective and the Kähler class is rational.

If it is canonically polarized, it carries a unique singular KE metric in the cohomology class of $K_{V}+\Delta$, regular outside $\Delta \cup V^{\text {sing }}$.

Furthermore, let $V^{o}$ be the largest open subset of $V^{\text {reg }}$ such that $\Delta \cap V^{o}$ has snc support and multiplicities of the form $1-\frac{1}{n}$ with $n \in \mathbb{N}^{*}$. Then, the singular KE metric becomes smooth on the stack $\left[V^{o}, \Delta \cap V^{o}\right]$.

Proof. For regularity on the smooth locus, we need the full statement of Theorems 3.5 and 4.5, poles included.

\section{ACKNOWLEDGMENTS}

The authors would like to thank Z. Blocki, S. Boucksom, A. Chiodo, J. P. Demailly, J. Keller, S. Kolodziej, M. Paun and B. Toën for useful conversations and C. Simpson and Y. T. Siu for inspiring remarks. We are grateful to the referee for his penetrating remarks.

\section{REFERENCES}

[A] T. Aubin: Équations du type Monge-Ampère sur les variétés kählériennes compactes. Bull. Sci. Math. (2) 102 (1978), no. 1, 63-95. MR494932 (81d:53047)

[BT] E. Bedford \& B. A. Taylor: A new capacity for plurisubharmonic functions. Acta Math. 149 (1982), no. 1-2, 1-40. MR674165 (84d:32024)

$[\mathrm{BM}] \quad$ E. Bierstone \& P. Milman: Canonical desingularization in characteristic zero by blowing up the maximum strata of a local invariant, Inv. Math. 128 (1997), 207-302. MR.1440306 (98e:14010)

[BCHM] C. Birkar, P. Cascini, C. D. Hacon, J. Mckernan: Existence of minimal models for varieties of log general type, arXiv:math/0610203.

[B1] Z. Blocki: Uniqueness and stability for the Monge-Ampère equation on compact Kaehler manifolds. Indiana Univ. Math. J. 52 (2003), no. 6, 1697-1701. MR2021054 (2004m:32073)

[B2] Z. Blocki: The Monge-Ampère equation on compact Kähler manifolds. Lecture Notes of a course given at the Winter School in Complex Analysis, Toulouse, 24-328/02/2005.

[BK] Z. Blocki \& S. Kolodziej: Regularization of plurisubharmonic functions on manifolds. Proc. Amer. Math. Soc. 135 (2007), no. 7, 2089-2093 . MR2299485 (2008a:32029)

[BGK] C. Boyer \& K. Galicki \& J. Kollár: Einstein metrics on spheres, Ann. Math. 162 (2005), 557-580. MR.2178969 (2006j:53058)

[Ca] E. Calabi: On Kähler manifolds with vanishing canonical class. Algebraic geometry and topology. A symposium in honor of S. Lefschetz, pp. 78-89. Princeton Univ. Press, Princeton, N.J. (1957). MR0085583(19:62b)

[CN] P. Cascini \& G. La Nave: Kähler-Ricci Flow and the Minimal Model Program for Projective Varieties. Preprint arXiv math. AG/0603064.

[Ce] U. Cegrell: Pluricomplex energy. Acta Math. 180 (1998), no. 2, 187-217. MR1638768 (99h:32016)

[Cor] A. Corti \& F. Ambro \& O. Fujino \& C. Hacon \& J. Kollár \& J. Mc Kernan \& H. Takagi: Extension theorems and the existence of flips, Flips for 3-folds and 4-folds (Alessio Corti, ed.), Oxford University Press (2007). MR2352762 (2008j:14031) 
[Deb] O. Debarre: Higher-dimensional algebraic geometry. Universitext. Springer-Verlag, New York (2001). xiv+233 pp. MR 1841091 (2002g:14001)

[Dem1] J. -P. Demailly: Monge-Ampère operators, Lelong numbers and intersection theory. Complex analysis and geometry, 115-193, Univ. Ser. Math., Plenum, New York (1993). MR.1211880 (94k:32009)

[Dem2] J. -P. DEMAILLY: A numerical criterion for very ample line bundles. J. Differential Geom. 37 (1993), no. 2, 323-374. MR1205448 (94d:14007)

[Dem3] J. -P. Demailly: Cohomology of $q$-convex spaces in top degrees, Math. Zeitschrift 203 (1990) 283-295. MR 1055992 (91e:32014)

[DP] J. -P. Demailly \& M. Paun: Numerical characterization of the Kähler cone of a compact Kähler manifold, Ann. of Math. 159 (2004), 1247-1274. MR2113021 (2005i:32020)

[FN] J. E. Fornaess \& R. Narasimhan: The Levi problem on complex space with singularities. Math. Ann. 248 (1980), 47-72. MR569410 (81f:32020)

[GR] H. Grauert \& R. Remmert: Coherent analytic sheaves. Grundlehren der Mathematischen Wissenschaften, 265. Springer-Verlag, Berlin (1984) xviii+249 pp. MR755331 (86a:32001)

[G] V. Guedj: Approximation of currents on complex manifolds. Math. Ann. 313 (1999), no. 3, 437-474. MR.1678537(2000b:32017)

[GZ1] V. Guedj \& A. Zeriahi: Intrinsic capacities on compact Kähler manifolds. J. Geom. Anal. 15 (2005), no. 4, 607-639. MR2203165 (2006j:32041)

[GZ2] V. Guedj \& A. Zeriahi: The weighted Monge-Ampère energy of quasiplurisubharmonic functions, J. Funct. Anal. 250 (2007), no. 2, 442-482. MR2352488 (2008h:32056)

[HMcK] C. Hacon \& J. Mckernan: On the existence of flips, math. AG/0507597; see also Extension theorems and the existence of flips, in Flips for 3-folds and 4-folds (Alessio Corti, ed.), Oxford University Press (2007), pp. 79-100. MR2359343

[Ha1] R. Hartshorne: Algebraic geometry. Graduate Texts in Mathematics, 52. SpringerVerlag, New York-Heidelberg (1977). xvi+496 pp. MR0463157 (57:3116)

[Ha2] R. Hartshorne: Stable reflexive sheaves. Math. Ann. 254 (1980), no. 2, 121-176. MR.597077 (82b:14011)

[Hi] H. Hironaka, Resolution of singularities over a field of characteristic zero. I,II. Annals of Math. 79 (1964), 109-326. MR0199184(33:7333)

[Hö] L. Hörmander: Notions of convexity, Birkhäuser (1994). MR1301332 (95k:00002)

$[\mathrm{Hu}] \quad$ A. T. Huckleberry: Subvarieties of homogeneous and almost homogeneous manifolds. Contributions to complex analysis and analytic geometry, 189-232, Aspects Math., E26, Vieweg, Braunschweig, 1994. MR1319349(96f:32061)

[Ka] Y. Kawamata: On the finiteness of generators of a pluricanonical ring for a 3-fold of general type. Amer. J. Math. 106 (1984), no. 6, 1503-1512. MR765589 (86j:14032)

[Kö] R. Kobayashi: Einstein-Kähler V-metrics on open Satake V-surfaces with isolated quotient singularities. Mathematische Annalen 272 (1985), 385-398. MR799669 (87b:32050)

[KM] J. Kollár \& S. Mori: Birational geometry of algebraic varieties. With the collaboration of C. H. Clemens and A. Corti. Translated from the 1998 Japanese original. Cambridge Tracts in Mathematics, 134. Cambridge University Press, Cambridge, 1998. viii+254 pp. MR1658959 (2000b:14018)

[K1] S. Kolodziej: The complex Monge-Ampère equation. Acta Math. 180 (1998), no. 1, 69-117. MR 1618325 (99h:32017)

[K2] S. Kolodziej: The Monge-Ampère equation on compact Kähler manifolds. Indiana Univ. Math. J. 52 (2003), no. 3, 667-686 MR1986892(2004i:32062)

[K3] S. Kolodziej: The complex Monge-Ampère equation and pluripotential theory. Mem. Amer. Math. Soc. 178 (2005), no. 840, x+64 pp. MR2172891 (2006d:32054)

[MO] K. Matsuki \& M. Olsson: Kawamata-Viehweg as Kodaira vanishing for stacks. Math. Res. Letters 12 (2005), 207-217. . MR2150877|(2006c:14023)

[Mo] S. Mori: Flip theorem and the existence of minimal models for 3-folds. J. Amer. Math. Soc. 1 (1988), no. 1, 117-253. MR924704(89a:14048)

[Na] M. Nakamaye: Stable base loci of linear series, Math. Ann. 318 (2000), 837-847. MR:1802513 (2002a:14008)

[Pa] M. Paun: Sur l'effectivité numérique des images inverses de fibrés en droites, Math. Ann. 310 (1998) (3), 411-421. MR1612321 (99c:32042) 
[R1] M. Reid: Canonical 3-folds. Journées de Géométrie Algébrique d'Angers, Juillet 1979, pp. 273-310, Sijthoff \& Noordhoff, Alphen aan den Rijn-Germantown, Md. (1980). MR605348(82i:14025)

[R2] M. Reid: Young person's guide to canonical singularities. Algebraic geometry, Bowdoin, 1985 (Brunswick, Maine, 1985), 345-414, Proc. Sympos. Pure Math., 46, Part 1, Amer. Math. Soc., Providence, RI (1987). MR.927963 (89b:14016)

[R] R. Richberg: Stetige streng pseudokonvexe Funktionen, Math. Ann. 175 (1968), 257286. MR 0222334 (36:5386)

[Sho] V. Shokurov: Prelimiting flips. Tr. Math. Inst. Steklova 240 (2003), 82-219. MR:1993750 (2004k:14024)

[S] N. Sibony: Dynamique des applications rationnelles de $\mathbf{P}^{k}$. Dynamique et géométrie complexes (Lyon, 1997), ix-x, xi-xii, 97-185, Panor. Synthèses, 8, Soc. Math. France, Paris (1999). MR1760844 (2001e:32026)

[Siu] Y. T. Siu: A vanishing theorem for semipositive line bundles over non-Kähler manifolds. J. Differential Geom. 19 (1984), no. 2, 431-452. MR755233 (86c:32029)

[Siu2] Y. T. Siu: Multiplier ideal sheaves in complex and algebraic geometry. Sci. China Ser. A 48 (2005), suppl., 1-31. MR2156488 (2007a:32023)

[ST] J. Song \& G. Tian: The Kähler-Ricci flow on surfaces of positive Kodaira dimension. Inv. Math. 170 (2007), 609-653. MR2357504

[Sg] K. Sugiyama: Einstein-Kähler Metrics on Minimal Varieties of General Type, in Recent Topics in Differential and Analytic Geometry, T. Ochiai, ed., Adv. Stud. in Pure Math. 18-I (1990), 417-443. MR.1145268 (93b:32047)

[T] G. Tian: Canonical metrics in Kähler geometry. Lectures in Mathematics ETH Zürich. Birkhäuser Verlag, Basel (2000). MR1787650(2001j:32024)

[TZ] G. Tian \& Z. Zhang: On the Kähler-Ricci flow of projective manifolds of general type. Chin. Ann. Math. 27 (2006), no. 2, 179-192. MR2243679 (2007c:32029)

[Ts] H. Tsuji: Existence and degeneration of Kähler-Einstein metrics on minimal algebraic varieties of general type. Math. Ann. 281 (1988), no. 1, 123-133. MR944606 (89e:53075)

[Y] S. T. Yau: On the Ricci curvature of a compact Kähler manifold and the complex Monge-Ampère equation. I. Comm. Pure Appl. Math. 31 (1978), no. 3, 339-411. MR 480350 (81d:53045)

[Z] A. Zeriahi: Volume and capacity of sublevel sets of a Lelong class of plurisubharmonic functions. Indiana Univ. Math. J. 50 (2001), no. 1, 671-703. MR1857051(2002f:32059)

[Zha] Z. Zhang: On Degenerated Monge-Ampere Equations over Closed Kähler Manifolds, Int. Math. Res. Not. (2006), Art. ID 63640, 18 pp. MR2233716 (2007b:32058)

Institut Fourier - UMR5582, 100 Rue des Maths, BP 74, 38402 St Martin D’Heres, FRANCE

E-mail address: eyssi@fourier.ujf-grenoble.fr

LATP, UMr 6632, CMi, Université de Provence, 39 Rue Joliot-Curie, 13453 Marseille CEDEX 13, FRANCE

E-mail address: guedj@cmi.univ-mrs.fr

Laboratoire Emile Picard, UMr 5580, Université Paul Sabatier, 118 route de NarBonne, 31062 Toulouse Cedex 04, France

E-mail address: zeriahi@math.ups-tlse.fr 